\title{
Excited bottom-charmed mesons in a nonrelativistic quark model
}

\author{
Qi Li, Ming-Sheng Liu, Long-Sheng Lu, Qi-Fang Lü, ${ }^{*}$ Long-Cheng Gui, ${ }^{\dagger}$ and Xian-Hui Zhong \\ Department of Physics, Hunan Normal University, Changsha 410081, China, \\ Synergetic Innovation Center for Quantum Effects and Applications (SICQEA), \\ Changsha 410081, China and Key Laboratory of Low-Dimensional Quantum Structures \\ and Quantum Control of Ministry of Education, Changsha 410081, China
}

(Received 1 April 2019; published 22 May 2019)

\begin{abstract}
Using the newly measured masses of $B_{c}(1 S)$ and $B_{c}(2 S)$ from the CMS Collaboration and the $1 S$ hyperfine splitting determined from the lattice QCD as constraints, we calculate the $B_{c}$ mass spectrum up to the $6 S$ multiplet with a nonrelativistic linear potential model. Furthermore, using the wave functions from this model we calculate the radiative transitions between the $B_{c}$ states within a constituent quark model. For the higher mass $B_{c}$ states lying above $D B$ threshold, we also evaluate the Okubo-Zweig-Iizuka (OZI) allowed two-body strong decays with the ${ }^{3} P_{0}$ model. Our study indicates that there is large potential for the observations of the low-lying $B_{c}$ states below the $D B$ threshold via their radiative transitions; in addition, some higher mass $B_{c}$ states, such as $B_{c}\left(2^{3} P_{2}\right), B_{c}\left(2^{3} D_{1}\right), B_{c}\left(3^{3} D_{1}\right), B_{c}\left(4^{3} P_{0}\right)$, and the $1 F$-wave $B_{c}$ states, might be first observed in their dominant strong decay channels $D B, D B^{*}$, or $D^{*} B$ at the LHC for their relatively narrow widths.
\end{abstract}

DOI: 10.1103/PhysRevD.99.096020

\section{INTRODUCTION}

The $B_{c}$ states are composed of a bottom-charmed quarkantiquark pair, as an important family of hadron spectra was predicted in theory about 40 years ago [1]; however, the experimental progress towards establishing the $B_{c}$ spectrum is not obvious. Except for the ground state $B_{c}$ meson observed in 1998 by the CDF Collaboration at Fermilab [2], until 2018, only the ATLAS Collaboration reported evidence of an excited $B_{c}$ state with a mass of $6842 \pm$ $9 \mathrm{MeV}$ [3] consistent with the values predicted for $B_{c}(2 S)$, while it was not confirmed by the LHCb Collaboration by using their $8 \mathrm{TeV}$ data sample [4]. The poor situation of the observations and measurements of the $B_{c}$ spectrum is due to the production yields being significantly smaller than those of the charmonium and bottomonium $(c \bar{c}$ and $b \bar{b})$ states. Fortunately, the LHC provides good opportunities for our search for the excited $B_{c}$ states with its high collision energies and integrated luminosity. Very recently, two excited $B_{c}^{+}$states were observed in the $B_{c}^{+} \pi^{+} \pi^{-}$invariant mass spectrum by the CMS Collaboration [5]. Signals are

\footnotetext{
*lvqifang@hunnu.edu.cn † guilongcheng@hunnu.edu.cn zhongxh@hunnu.edu.cn
}

Published by the American Physical Society under the terms of the Creative Commons Attribution 4.0 International license. Further distribution of this work must maintain attribution to the author(s) and the published article's title, journal citation, and DOI. Funded by SCOAP ${ }^{3}$. consistent with the $B_{c}(2 S)$ and $B_{c}^{*}(2 S)$ states. These two states are well resolved from each other and are observed with a significance exceeding five standard deviations. The mass of the $B_{c}(2 S)$ meson, $6871 \pm 2.8 \mathrm{MeV}$, measured by the CMS Collaboration is inconsistent with the determination $6842 \pm 9 \mathrm{MeV}$ by the ATLAS Collaboration. The reason is that the peak observed by ATLAS could be the superposition of the $B_{c}(2 S)$ and $B_{c}^{*}(2 S)$ states, too closely spaced with respect to the resolution of the measurement [5].

The $B_{c}$ states as the only conventional heavy mesons with different flavors have aroused great interest in theory. Compared with the $c \bar{c}$ and $b \bar{b}$ spectra, the $B_{c}$ spectrum has several special features for the bottom-charmed quarkantiquark pair. (i) The $B_{c}$ states cannot annihilate into gluons; thus, the low-lying excited $B_{c}$ states below the $D B$ threshold are more stable with a narrow width less than a few hundred $\mathrm{keV}$, and they mainly decay via the electromagnetic or hadronic transitions between two different $B_{c}$ states. (ii) In the $B_{c}$ meson spectrum there are configuration mixings between the states with different total spins but with the same total angular momentum, such as ${ }^{3} P_{1}-{ }^{1} P_{1}$, ${ }^{3} D_{2}-{ }^{1} D_{2}$, and ${ }^{3} F_{3}-{ }^{1} F_{3}$ mixings via the antisymmetric part of the spin-orbit potential. (iii) Additionally, the $B_{c}$ states provide a unique window for studying the heavyquark dynamics that is very different from those provided by the $c \bar{c}$ and $b \bar{b}$ states. In the past years, the $B_{c}$ mass spectrum has been predicted with various models [6-34]. Furthermore, a few lattice calculations can be found in Refs. [35-39]. To estimate the production rates in experiments, the production of the excited $B_{c}$ states was often 
discussed in the literature [40-53]. As the dominant decay modes, the electromagnetic transitions of the low-lying $B_{c}$ states were also widely estimated in the literature [7-16, 54-58]. However, the studies of the Okubo-Zweig-Iizuka (OZI)-allowed strong decays for the high-lying $B_{c}$ states are confined only to a few calculations $[17,18,59,60]$.

The successes of the observations of the radially excited $B_{c}$ states $B_{c}(2 S)$ and $B_{c}^{*}(2 S)$ by the CMS Collaboration [5] have demonstrated that more excited $B_{c}$ states are to be discovered in future LHC experiments. Stimulated by the great discovery potentials of the missing $B_{c}$ states in future experiments, in the present work we carry out a systematic study of the $B_{c}$ spectrum. First, using the newly measured masses of $B_{c}(1 S)$ and $B_{c}(2 S)$ from the CMS Collaboration [5] and the $1 S$ hyperfine splitting determined from the lattice QCD [36-38] as constraints, we calculate the $B_{c}$ mass spectrum up to the $6 S$ multiplet with a nonrelativistic linear potential model. The slope parameter of the linear potential has been well determined in our previous study of the charmonium states [61]. To involve the spin-dependent corrections of the spatial wave functions, following the method adopted in Refs. [61,62], we treat the spin-dependent interactions as nonperturbative terms in our calculations. With this nonperturbative treatment, we can reasonably include the effect of spindependent interactions on the spatial wave functions, which is essential for us to gain reliable predictions of the decay behaviors.

Then, with the available wave functions from the potential model, we evaluate the electromagnetic (EM) transitions between the $B_{c}$ states within a nonrelativistic constituent quark model developed in our previous works [61,62]. With this approach, the possible higher EM multipole contributions to an EM transition process can be included naturally. Considering the fact that the higher $B_{c}$ states lying above the $D B$ threshold may have enough possibilities to be produced at LHC, and they are easy to be established in the $D^{(*)} B^{(*)}$ hadronic final states, thus, to give useful references for the LHC observations, we further calculate the OZI-allowed strong decays of the higher $B_{c}$ states within the widely used ${ }^{3} P_{0}$ model [63-65]. It is found that $B_{c}\left(2^{3} P_{2}\right), B_{c}\left({ }^{3} D_{1}\right), B_{c}\left(3^{3} D_{1}\right)$ together with the $1 F$-wave $B_{c}$ states might be first observed in their dominant strong decay channels $D B, D B^{*}$, or $D^{*} B$ at $\mathrm{LHC}$ for their relatively narrow width.

This paper is organized as follows. In Sec. II, the $B_{c}$ mass spectrum is calculated within a nonrelativistic linear potential model. Then, with the obtained $B_{c}$ spectrum the radiative transitions between the $B_{c}$ states are estimated in Sec. III within a nonrelativistic constituent quark model. In Sec. IV, the OZI-allowed two-body strong decays of the excited $B_{c}$ state are also studied within the ${ }^{3} P_{0}$ model. In Sec. V, we focus on the calculation results and discuss some strategies for looking for the $B_{c}$ states in future experiments. Finally, a summary is given in Sec. VI.

\section{MASS SPECTRUM}

To describe a bottom-charmed meson system, we adopt a nonrelativistic linear potential model. In this model, the effective quark-antiquark potential is written as the sum of the spin-independent term $H_{0}(r)$ and spin-dependent term $H_{s d}(r)$; i.e.,

$$
V(r)=H_{0}(r)+H_{s d}(r),
$$

where

$$
H_{0}(r)=-\frac{4}{3} \frac{\alpha_{s}}{r}+b r
$$

includes the standard color Coulomb interaction and the linear confinement. The spin-dependent part $H_{s d}(r)$ can be expressed as $[1,9,11]$

$$
H_{s d}(r)=H_{S S}+H_{T}+H_{L S},
$$

where

$$
H_{S S}=\frac{32 \pi \alpha_{S}}{9 m_{q} m_{\bar{q}}} \tilde{\delta}_{\sigma}(r) \mathbf{S}_{q} \cdot \mathbf{S}_{\bar{q}}
$$

is the spin-spin contact hyperfine potential. Here, we take $\tilde{\delta}_{\sigma}(r)=(\sigma / \sqrt{\pi})^{3} e^{-\sigma^{2} r^{2}}$ as suggested in Ref. [66]. The tensor potential $H_{T}$ is adopted as

$$
H_{T}=\frac{4}{3} \frac{\alpha_{s}}{m_{q} m_{\bar{q}}} \frac{1}{r^{3}}\left(\frac{3 \mathbf{S}_{q} \cdot \mathbf{r} \mathbf{S}_{\bar{q}} \cdot \mathbf{r}}{r^{2}}-\mathbf{S}_{q} \cdot \mathbf{S}_{\bar{q}}\right)
$$

For convenience in the calculations, the potential of the spin-orbit interaction $H_{L S}$ is decomposed into symmetric part $H_{\text {sym }}$ and antisymmetric part $H_{\text {anti }}$,

$$
H_{L S}=H_{\text {sym }}+H_{\text {anti }},
$$

with

$$
\begin{gathered}
H_{\mathrm{sym}}=\frac{\mathbf{S}_{+} \cdot \mathbf{L}}{2}\left[\left(\frac{1}{2 m_{\bar{q}}^{2}}+\frac{1}{2 m_{q}^{2}}\right)\left(\frac{4}{3} \frac{\alpha_{s}}{r^{3}}-\frac{b}{r}\right)+\frac{8 \alpha_{s}}{3 m_{q} m_{\bar{q}} r^{3}}\right], \\
H_{\text {anti }}=\frac{\mathbf{S}_{-} \cdot \mathbf{L}}{2}\left(\frac{1}{2 m_{q}^{2}}-\frac{1}{2 m_{\bar{q}}^{2}}\right)\left(\frac{4}{3} \frac{\alpha_{s}}{r^{3}}-\frac{b}{r}\right) .
\end{gathered}
$$

In these equations, $\mathbf{L}$ is the relative orbital angular momentum of the $q \bar{q}$ system; $\mathbf{S}_{q}$ and $\mathbf{S}_{\bar{q}}$ are the spins of the quark $q$ and antiquark $\bar{q}$, respectively, and $\mathbf{S}_{ \pm} \equiv \mathbf{S}_{q} \pm$ $\mathbf{S}_{\bar{q}} ; m_{q}$ and $m_{\bar{q}}$ are the masses of quark $q$ and antiquark $\bar{q}$, respectively; $\alpha_{s}$ is the running coupling constant of QCD; and $r$ is the distance between the quark $q$ and antiquark $\bar{q}$. 
The five parameters in the above equations $\left(\alpha_{s}, b, \sigma, m_{b}\right.$, $m_{c}$ ) are determined by fitting the spectrum.

We can get the masses and wave functions by solving the radial Schrödinger equation,

$$
\frac{d^{2} u(r)}{d r^{2}}+2 \mu_{R}\left[E-V_{q \bar{q}}(r)-\frac{L(L+1)}{2 \mu_{R} r^{2}}\right] u(r)=0,
$$

with

$$
V_{q \bar{q}}(r)=V(r)+H_{S S}+H_{S L}+H_{T},
$$

where $\mu_{R}=m_{q} m_{\bar{q}} /\left(m_{q}+m_{\bar{q}}\right)$ is the reduced mass of the system, and $E$ is the binding energy of the system. Then, the mass of a bottom-charmed state is obtained by

$$
M_{q \bar{q}}=m_{q}+m_{\bar{q}}+E .
$$

In this work, to reasonably include the corrections from these spin-dependent potentials to both the mass and wave function of a meson state, we deal with the spindependent interactions nonperturbatively. We solve the radial Schrödinger equation by using the three-point difference central method [67] from central $(r=0)$ towards outside $(r \rightarrow \infty)$ point by point. This method was successfully to deal with the spectroscopies of $c \bar{c}$ and $b \bar{b}[61,62]$. To overcome the singular behavior of $1 / r^{3}$ in the spin-dependent potentials, following the method of our previous works $[61,62]$, we introduce a cutoff distance $r_{c}$ in the calculation. Within a small range $r \in\left(0, r_{c}\right)$, we let $1 / r^{3}=1 / r_{c}^{3}$.

Finally, it should be mentioned that the antisymmetric part of the spin-orbit potential, $H_{\text {anti }}$, can let the states with different total spins but with the same total angular momentum, such as $B_{c}\left(n^{3} L_{J}\right)$ and $B_{c}\left(n^{1} L_{J}\right)$, mix with each other. Thus, as mixing states between $B_{c}\left(n^{3} L_{J}\right)$ and $B_{c}\left(n^{1} L_{J}\right)$, the physical $B_{c}$ states $B_{c}(n L)$ and $B_{c}\left(n L^{\prime}\right)$ are expressed as

$$
\left(\begin{array}{l}
B_{c}\left(n L_{J}^{\prime}\right) \\
B_{c}\left(n L_{J}\right)
\end{array}\right)=\left(\begin{array}{cc}
\cos \theta_{n L} & \sin \theta_{n L} \\
-\sin \theta_{n L} & \cos \theta_{n L}
\end{array}\right)\left(\begin{array}{c}
B_{c}\left(n^{1} L_{J}\right) \\
B_{c}\left(n^{3} L_{J}\right)
\end{array}\right),
$$

where $J=L=1,2,3 \cdots$, and the $\theta_{n L}$ is the mixing angle. In this work $B_{c}\left(n L^{\prime}\right)$ corresponds to the higher mass mixed state as often adopted in the literature.

In this work the parameter set is taken as $\alpha_{s}=0.5021$, $b=0.1425 \mathrm{GeV}^{2}, m_{b}=4.852 \mathrm{GeV}, m_{c}=1.483 \mathrm{GeV}$, $\sigma=1.3 \mathrm{GeV}$, and $r_{c}=0.16 \mathrm{fm}$. To be consistent with our previous study [61], the charmed quark mass $m_{c}$ and the slope for the linear confining potential are taken from the determinations, i.e., $m_{c}=1.483 \mathrm{GeV}$ and $b=$ $0.1425 \mathrm{GeV}^{2}$. The other three parameters $\left(m_{b}, \alpha_{s}, \sigma\right)$ are determined by fitting the masses of the $B_{c}, B_{c}^{*}$, and $B_{c}(2 S)$ mesons. The masses of $B_{c}$ and $B_{c}(2 S)$ are taken from the recent measurements of the CMS Collaboration [5]. Although the $B_{c}^{*}$ meson is still not measured in experiments, the mass difference between the $B_{c}^{*}$ and $B_{c}$ is predicted to be around $55 \mathrm{MeV}$ from lattice QCD [36-38]. Thus, combining it with the measured mass $6271 \mathrm{MeV}$ for $B_{c}$, in present work we estimate the mass of $B_{c}^{*}$ as $\sim 6326 \mathrm{MeV}$. The cutoff distance $r_{c}$ is determined by the mass of $B_{c}\left(1^{3} P_{0}\right)$. To determine the mass of $B_{c}\left(1^{3} P_{0}\right)$, we adopt a method of perturbation; i.e., we let $H=H_{0}+H^{\prime}$, where $H^{\prime}$ is a part which contains the term of $1 / r^{3}$. By solving the equation of $H_{0}\left|\psi_{n}^{(0)}\right\rangle=E_{0}\left|\psi_{n}^{(0)}\right\rangle$, we can get the energy $E_{0}$ and wave function $\left|\psi_{n}^{(0)}\right\rangle$; then, we obtain the mass of $B_{c}\left(1^{3} P_{0}\right), M=m_{b}+m_{c}+E_{0}+\left\langle\psi_{n}^{(0)}\left|H^{\prime}\right| \psi_{n}^{(0)}\right\rangle$.

By solving the radial Schrödinger equation and with the determined parameter set, we obtain the masses of the bottom-charmed states, which have been listed in Table I and shown in Fig. 1. For comparison, the other model predictions in Refs. $[7-11,15,16]$ are listed in the same table as well.

It is found that the masses of the low-lying $1 S-, 2 S-, 3 S_{-}$, $1 P$-, $2 P-, 1 D$-wave $B_{c}$ states predicted in this work are compatible with the other potential model predictions. For the higher mass states, such as $4 S_{-}, 5 S_{-}, 6 S_{-}, 3 P_{-}, 4 P_{-}, 2 D_{-}$, $2 F-, 3 F$-wave states, the masses predicted by us are very close to those predicted with a relativistic model in Ref. [8], while they are about 100-200 MeV smaller than those predicted in Refs. [15,16]. Furthermore, the hyperfine splitting between $B_{c}^{*}(2 S)$ and $B_{c}(2 S)$ is predicted to be $19 \mathrm{MeV}$, which is slightly smaller than $30-45 \mathrm{MeV}$, predicted in previous works [7-11,15,16,36-38]. Finally, it should be pointed out that the mixing angles for ${ }^{3} P_{1}-{ }^{1} P_{1},{ }^{3} D_{2}-{ }^{1} D_{2}$, and ${ }^{3} F_{3}-{ }^{1} F_{3}$ have obvious model dependencies (see Table II).

\section{RADIATIVE TRANSITIONS}

We use the nonrelativistic constituent quark model as adopted in Refs. [61,62,68-72] to calculate the radiative transitions between the $B_{c}$ states. In this model, the quarkphoton EM coupling at the tree level is taken as

$$
H_{e}=-\sum_{j} e_{j} \bar{\psi}_{j} \gamma_{\mu}^{j} A^{\mu}(\mathbf{k}, \mathbf{r}) \psi_{j}
$$

where $A^{\mu}$ represents the photon field with three momenta $\mathbf{k}$; while $e_{j}$ and $\mathbf{r}_{j}$ stand for the charge and coordinate of the constituent quark $\psi_{j}$, respectively. In order to match the nonrelativistic wave functions of the $B_{c}$ states, we adopt the nonrelativistic form of Eq. (13), which is given by [73-78]

$$
H_{e}^{n r}=\sum_{j}\left[e_{j} \mathbf{r}_{j} \cdot \boldsymbol{\epsilon}-\frac{e_{j}}{2 m_{j}} \boldsymbol{\sigma}_{j} \cdot(\boldsymbol{\epsilon} \times \hat{\mathbf{k}})\right] e^{-i \mathbf{k} \cdot \mathbf{r}_{j}},
$$


TABLE I. Predicted masses $(\mathrm{MeV})$ of $B_{c}$ states compared with other model predictions and data. The mixing angles between $B_{c}\left(n^{3} L_{J}\right)$ and $B_{c}\left(n^{1} L_{J}\right)$ obtained in this work are presented in Table II.

\begin{tabular}{|c|c|c|c|c|c|c|c|c|c|c|c|}
\hline State & $J^{P}$ & Ours & ZVR [8] & SJSCP [16] & MBV [15] & EQ [7] & EFG [10] & GI [11] & KLT [9] & Lattice [36] & Exp [5] \\
\hline$B_{c}\left(1^{3} S_{1}\right)$ & $1^{-}$ & 6326 (input) & 6340 & 6321 & 6357 & 6337 & 6332 & 6338 & 6317 & $6331 \pm 10$ & $\ldots$ \\
\hline$B_{c}\left(1^{1} S_{0}\right)$ & $0^{-}$ & 6271 (input) & 6260 & 6272 & 6275 & 6264 & 6270 & 6271 & 6253 & $6276 \pm 9$ & 6271 \\
\hline$B_{c}\left(2^{3} S_{1}\right)$ & $1^{-}$ & 6890 & 6900 & 6900 & 6897 & 6899 & 6881 & 6887 & 6902 & $\ldots$ & $\ldots$ \\
\hline$B_{c}\left(2{ }^{1} S_{0}\right)$ & $0^{-}$ & 6871 (input) & 6850 & 6864 & 6862 & 6856 & 6835 & 6855 & 6867 & & 6871 \\
\hline$B_{c}\left(3^{3} S_{1}\right)$ & $1^{-}$ & 7252 & 7280 & 7338 & 7333 & 7280 & 7235 & 7272 & $\ldots$ & $\cdots$ & $\ldots$ \\
\hline$B_{c}\left(3{ }^{1} S_{0}\right)$ & $0^{-}$ & 7239 & 7240 & 7306 & 7308 & 7244 & 7193 & 7250 & $\ldots$ & $\cdots$ & $\ldots$ \\
\hline$B_{c}\left(4^{3} S_{1}\right)$ & $1^{-}$ & 7550 & 7580 & 7714 & 7734 & 7594 & $\ldots$ & $\ldots$ & $\ldots$ & $\ldots$ & $\ldots$ \\
\hline$B_{c}\left(4^{1} S_{0}\right)$ & $0^{-}$ & 7540 & 7550 & 7684 & 7713 & 7562 & $\cdots$ & $\cdots$ & $\cdots$ & $\cdots$ & $\cdots$ \\
\hline$B_{c}\left(5^{3} S_{1}\right)$ & $1^{-}$ & 7813 & $\ldots$ & 8054 & 8115 & $\ldots$ & $\cdots$ & $\cdots$ & $\cdots$ & $\cdots$ & $\cdots$ \\
\hline$B_{c}\left(5^{1} S_{0}\right)$ & $0^{-}$ & 7805 & $\ldots$ & 8025 & 8097 & $\ldots$ & $\cdots$ & $\ldots$ & $\cdots$ & $\ldots$ & $\cdots$ \\
\hline$B_{c}\left(6^{3} S_{1}\right)$ & $1^{-}$ & 8054 & $\ldots$ & 8368 & 8484 & $\ldots$ & $\ldots$ & $\ldots$ & $\ldots$ & $\ldots$ & $\ldots$ \\
\hline$B_{c}\left(6^{1} S_{0}\right)$ & $0^{-}$ & 8046 & $\ldots$ & 8340 & 8469 & $\ldots$ & $\ldots$ & $\ldots$ & $\ldots$ & $\ldots$ & $\ldots$ \\
\hline$B_{c}\left(1^{3} P_{2}\right)$ & $2^{+}$ & 6787 & 6760 & 6712 & 6737 & 6747 & 6762 & 6768 & 6743 & $\ldots$ & $\ldots$ \\
\hline$B_{c}\left(1 P_{1}^{\prime}\right)$ & $1^{+}$ & 6776 & 6740 & $\ldots$ & 6734 & 6736 & 6749 & 6750 & 6729 & $\ldots$ & $\ldots$ \\
\hline$B_{c}\left(1 P_{1}\right)$ & $1^{+}$ & 6757 & 6730 & $\ldots$ & 6686 & 6730 & 6734 & 6741 & 6717 & $6736 \pm 24$ & $\cdots$ \\
\hline$B_{c}\left(1^{3} P_{0}\right)$ & $0^{+}$ & 6714 & 6680 & 6686 & 6638 & 6700 & 6699 & 6706 & 6683 & $6712 \pm 25$ & $\cdots$ \\
\hline$B_{c}\left(2^{3} P_{2}\right)$ & $2^{+}$ & 7160 & 7160 & 7173 & 7175 & 7153 & 7156 & 7164 & 7134 & $\ldots$ & $\cdots$ \\
\hline$B_{c}\left(2 P_{1}^{\prime}\right)$ & $1^{+}$ & 7150 & 7150 & $\ldots$ & 7173 & 7142 & 7145 & 7150 & 7124 & $\ldots$ & $\ldots$ \\
\hline$B_{c}\left(2 P_{1}\right)$ & $1^{+}$ & 7134 & 7140 & $\ldots$ & 7137 & 7135 & 7126 & 7145 & 7113 & $\cdots$ & $\cdots$ \\
\hline$B_{c}\left(2^{3} P_{0}\right)$ & $0^{+}$ & 7107 & 7100 & 7146 & 7084 & 7108 & 7091 & 7122 & 7088 & $\cdots$ & $\cdots$ \\
\hline$B_{c}\left(3^{3} P_{2}\right)$ & $2^{+}$ & 7464 & 7480 & 7565 & 7575 & $\ldots$ & $\ldots$ & $\ldots$ & $\ldots$ & $\cdots$ & $\cdots$ \\
\hline$B_{c}\left(3 P_{1}^{\prime}\right)$ & $1^{+}$ & 7458 & 7470 & $\ldots$ & 7572 & $\ldots$ & $\ldots$ & $\ldots$ & $\ldots$ & $\ldots$ & $\ldots$ \\
\hline$B_{c}\left(3 P_{1}\right)$ & $1^{+}$ & 7441 & 7460 & $\ldots$ & 7546 & $\cdots$ & $\ldots$ & $\cdots$ & $\ldots$ & $\cdots$ & $\ldots$ \\
\hline$B_{c}\left(3^{3} P_{0}\right)$ & $0^{+}$ & 7420 & 7430 & 7536 & 7492 & $\cdots$ & $\cdots$ & $\cdots$ & $\cdots$ & $\cdots$ & $\cdots$ \\
\hline$B_{c}\left(4^{3} P_{2}\right)$ & $2^{+}$ & 7732 & 7760 & 7915 & 7970 & $\ldots$ & $\ldots$ & $\ldots$ & $\ldots$ & $\ldots$ & $\ldots$ \\
\hline$B_{c}\left(4 P_{1}^{\prime}\right)$ & $1^{+}$ & 7727 & 7740 & $\ldots$ & 7942 & $\cdots$ & $\ldots$ & $\cdots$ & $\ldots$ & $\cdots$ & $\cdots$ \\
\hline$B_{c}\left(4 P_{1}\right)$ & $1^{+}$ & 7710 & 7740 & $\ldots$ & 7943 & $\cdots$ & $\cdots$ & $\cdots$ & $\cdots$ & $\cdots$ & $\cdots$ \\
\hline$B_{c}\left(4^{3} P_{0}\right)$ & $0^{+}$ & 7693 & 7710 & 7885 & 7970 & $\ldots$ & $\ldots$ & $\ldots$ & $\ldots$ & $\ldots$ & $\ldots$ \\
\hline$B_{c}\left(1^{3} D_{3}\right)$ & $3^{-}$ & 7030 & 7040 & 6990 & 7004 & 7005 & 7081 & 7045 & 7134 & $\ldots$ & $\ldots$ \\
\hline$B_{c}\left(1 D_{2}^{\prime}\right)$ & $2^{-}$ & 7032 & 7030 & $\ldots$ & 7003 & 7012 & 7079 & 7036 & 7124 & $\cdots$ & $\cdots$ \\
\hline$B_{c}\left(1 D_{2}\right)$ & $2^{-}$ & 7024 & 7020 & $\ldots$ & 6974 & 7009 & 7077 & 7041 & 7113 & $\ldots$ & $\ldots$ \\
\hline$B_{c}\left(1^{3} D_{1}\right)$ & $1^{-}$ & 7020 & 7010 & 6998 & 6973 & 7012 & 7072 & 7025 & 7088 & $\ldots$ & $\ldots$ \\
\hline$B_{c}\left(2^{3} D_{3}\right)$ & $3^{-}$ & 7348 & 7370 & 7399 & 7410 & $\ldots$ & $\ldots$ & $\ldots$ & $\ldots$ & $\cdots$ & $\cdots$ \\
\hline$B_{c}\left(2 D_{2}^{\prime}\right)$ & $2^{-}$ & 7347 & 7360 & $\ldots$ & 7408 & $\cdots$ & $\cdots$ & $\cdots$ & $\cdots$ & $\cdots$ & $\cdots$ \\
\hline$B_{c}\left(2 D_{2}\right)$ & $2^{-}$ & 7343 & 7360 & $\ldots$ & 7385 & $\ldots$ & $\cdots$ & $\ldots$ & $\cdots$ & $\ldots$ & $\ldots$ \\
\hline$B_{c}\left(2^{3} D_{1}\right)$ & $1^{-}$ & 7336 & 7350 & 7403 & 7377 & $\cdots$ & $\cdots$ & $\cdots$ & $\cdots$ & $\cdots$ & $\cdots$ \\
\hline$B_{c}\left(3^{3} D_{3}\right)$ & $3^{-}$ & 7625 & 7660 & 7761 & 7796 & $\cdots$ & $\cdots$ & $\cdots$ & $\cdots$ & $\cdots$ & $\cdots$ \\
\hline$B_{c}\left(3 D_{2}^{\prime}\right)$ & $2^{-}$ & 7623 & 7650 & $\ldots$ & 7783 & $\cdots$ & $\ldots$ & $\cdots$ & $\ldots$ & $\cdots$ & $\cdots$ \\
\hline$B_{c}\left(3 D_{2}\right)$ & $2^{-}$ & 7620 & 7650 & $\ldots$ & 7781 & $\cdots$ & $\cdots$ & $\cdots$ & $\cdots$ & $\cdots$ & $\cdots$ \\
\hline$B_{c}\left(3^{3} D_{1}\right)$ & $1^{-}$ & 7611 & 7640 & 7762 & 7761 & $\cdots$ & $\cdots$ & $\ldots$ & $\cdots$ & $\cdots$ & $\cdots$ \\
\hline$B_{c}\left(1^{3} F_{4}\right)$ & $4^{+}$ & 7227 & 7250 & 7244 & $\ldots$ & $\ldots$ & $\ldots$ & 7271 & $\ldots$ & $\ldots$ & $\ldots$ \\
\hline$B_{c}\left(1 F_{3}^{\prime}\right)$ & $3^{+}$ & 7240 & 7250 & $\ldots$ & $\ldots$ & $\ldots$ & $\ldots$ & 7266 & $\ldots$ & $\ldots$ & $\ldots$ \\
\hline$B_{c}\left(1 F_{3}\right)$ & $3^{+}$ & 7224 & 7240 & $\ldots$ & $\ldots$ & $\ldots$ & $\ldots$ & 7276 & $\ldots$ & $\ldots$ & $\ldots$ \\
\hline$B_{c}\left(1^{3} F_{2}\right)$ & $2^{+}$ & 7235 & 7240 & 7234 & $\ldots$ & $\ldots$ & $\ldots$ & 7269 & $\ldots$ & $\cdots$ & $\cdots$ \\
\hline$B_{c}\left(2^{3} F_{4}\right)$ & $4^{+}$ & 7514 & 7550 & 7617 & $\cdots$ & $\cdots$ & $\cdots$ & 7568 & $\cdots$ & $\cdots$ & $\cdots$ \\
\hline$B_{c}\left(2 F_{3}^{\prime}\right)$ & $3^{+}$ & 7525 & 7550 & $\ldots$ & $\ldots$ & $\cdots$ & $\cdots$ & 7571 & $\cdots$ & $\ldots$ & $\ldots$ \\
\hline$B_{c}\left(2 F_{3}\right)$ & $3^{+}$ & 7508 & 7540 & $\cdots$ & $\cdots$ & $\cdots$ & $\cdots$ & 7563 & $\cdots$ & $\cdots$ & $\cdots$ \\
\hline$B_{c}\left(2^{3} F_{2}\right)$ & $2^{+}$ & 7518 & 7540 & 7607 & $\cdots$ & $\cdots$ & $\cdots$ & 7565 & $\cdots$ & $\cdots$ & $\cdots$ \\
\hline$B_{c}\left(3^{3} F_{4}\right)$ & $4^{+}$ & 7771 & 7810 & 7956 & $\cdots$ & $\cdots$ & $\cdots$ & $\ldots$ & $\cdots$ & $\cdots$ & $\cdots$ \\
\hline$B_{c}\left(3 F_{3}^{\prime}\right)$ & $3^{+}$ & 7779 & 7810 & $\ldots$ & $\cdots$ & $\cdots$ & $\cdots$ & $\cdots$ & $\cdots$ & $\ldots$ & $\ldots$ \\
\hline$B_{c}\left(3 F_{3}\right)$ & $3^{+}$ & 7768 & 7800 & $\ldots$ & $\ldots$ & $\cdots$ & $\cdots$ & $\ldots$ & $\cdots$ & $\cdots$ & $\cdots$ \\
\hline$B_{c}\left(3^{3} F_{2}\right)$ & $2^{+}$ & 7730 & 7800 & 7946 & $\cdots$ & $\ldots$ & $\cdots$ & $\ldots$ & $\cdots$ & $\cdots$ & $\ldots$ \\
\hline
\end{tabular}




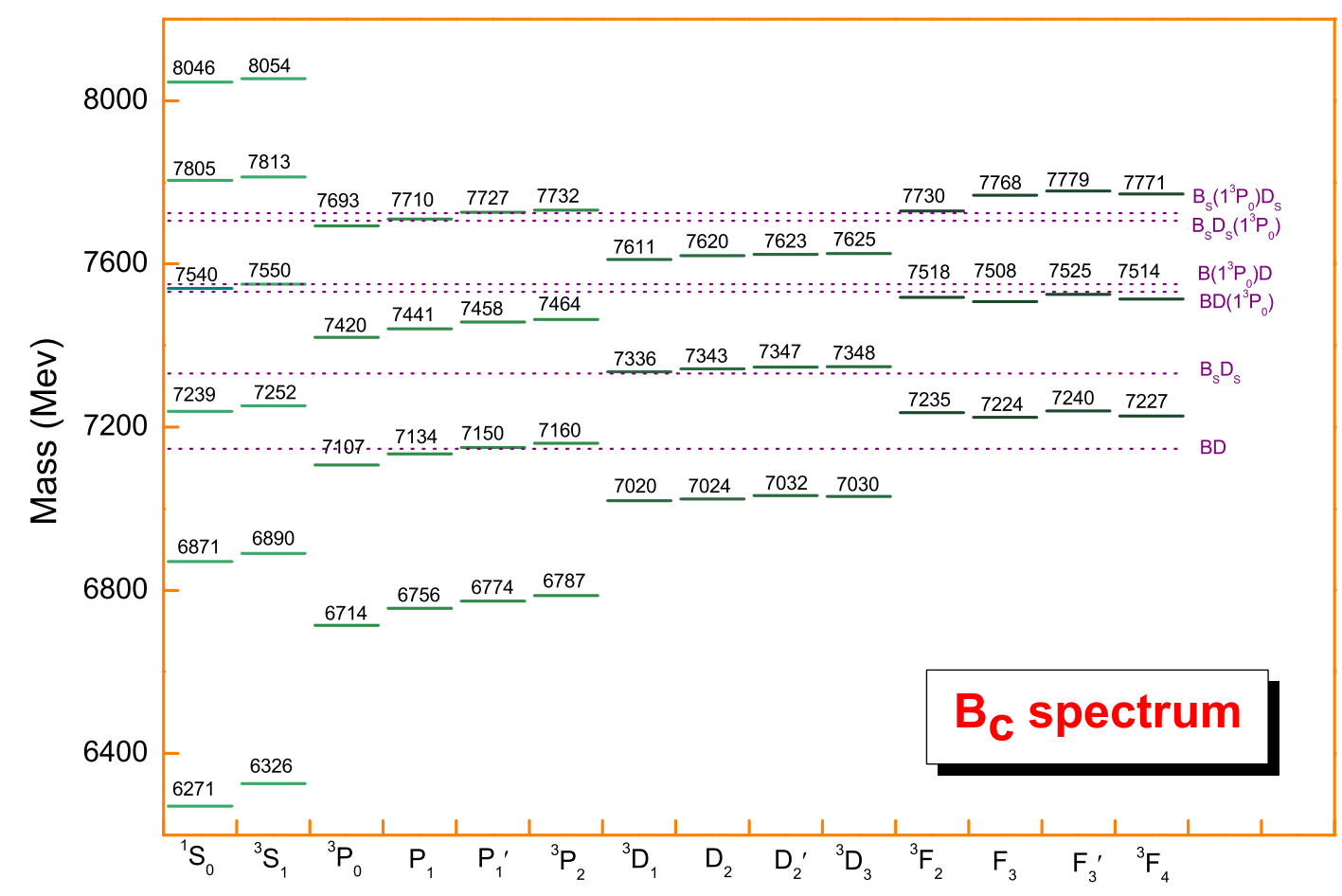

FIG. 1. The spectrum of $B_{c}$ mesons.

where $\boldsymbol{\epsilon}$ is the polarization vector of the final photon, $m_{j}$ and $\sigma_{j}$ stand for the constituent mass and Pauli spin vector for the $j$ th quark. The helicity amplitude $\mathcal{A}$ can be expressed as

$$
\mathcal{A}=-i \sqrt{\frac{\omega_{\gamma}}{2}}\left\langle f\left|H_{e}^{n r}\right| i\right\rangle
$$

Finally, we obtain the partial decay width of a radiative transition by

$$
\Gamma=\frac{|\mathbf{k}|^{2}}{\pi} \frac{2}{2 J_{i}+1} \frac{M_{f}}{M_{i}} \sum_{J_{f z}, J_{i z}}\left|\mathcal{A}_{J_{f z}, J_{i z}}\right|^{2},
$$

where $J_{i}$ is the total angular momentum of an initial meson, and $J_{f z}$ and $J_{i z}$ are the components of the total angular

TABLE II. Mixing angles.

\begin{tabular}{lcccc}
\hline \hline Mixing angle & Ours & {$[11]$} & {$[14]$} & {$[10]$} \\
\hline$\theta_{1 P}$ & $35.5^{\circ}$ & $22.4^{\circ}$ & $20.57^{\circ}$ & $20.4^{\circ}$ \\
$\theta_{2 P}$ & $38.0^{\circ}$ & $18.9^{\circ}$ & $19.94^{\circ}$ & $23.2^{\circ}$ \\
$\theta_{3 P}$ & $39.7^{\circ}$ & $\ldots$ & $17.68^{\circ}$ & $\ldots$ \\
$\theta_{4 P}$ & $39.7^{\circ}$ & $\ldots$ & $\ldots$ & $\ldots$ \\
$\theta_{1 D}$ & $45.0^{\circ}$ & $44.5^{\circ}$ & $-2.49^{\circ}$ & $-35.9^{\circ}$ \\
$\theta_{2 D}$ & $45.0^{\circ}$ & $\ldots$ & $-2.8^{\circ}$ & $\ldots$ \\
$\theta_{3 D}$ & $45.0^{\circ}$ & $\ldots$ & $\ldots$ & $\ldots$ \\
$\theta_{1 F}$ & $41.4^{\circ}$ & $41.4^{\circ}$ & $\ldots$ & $\ldots$ \\
$\theta_{2 F}$ & $43.4^{\circ}$ & $\ldots$ & $\ldots$ & $\ldots$ \\
$\theta_{3 F}$ & $42.4^{\circ}$ & $\ldots$ & $\ldots$ & $\ldots$ \\
\hline \hline
\end{tabular}

momenta along the $z$ axis of initial and final mesons, respectively. $M_{i}$ and $M_{f}$ correspond to the masses of the initial and final $B_{c}$ states, respectively.

The radiative decay properties for the $B_{c}$ states have been listed in Tables III-VIII. For comparison, some other predictions of the low-lying $B_{c}$ states from Refs. [7,9-11] are also given in the tables.

\section{STRONG DECAYS}

In this work, we use the ${ }^{3} P_{0}$ model [63-65] to calculate the OZI-allowed strong decays of the bottom-charmed

TABLE III. Partial widths of the $M 1$ transitions for the lowlying $1 S$-, $2 S$-, and $3 S$-wave $B_{c}$ states compared with the other model predictions.

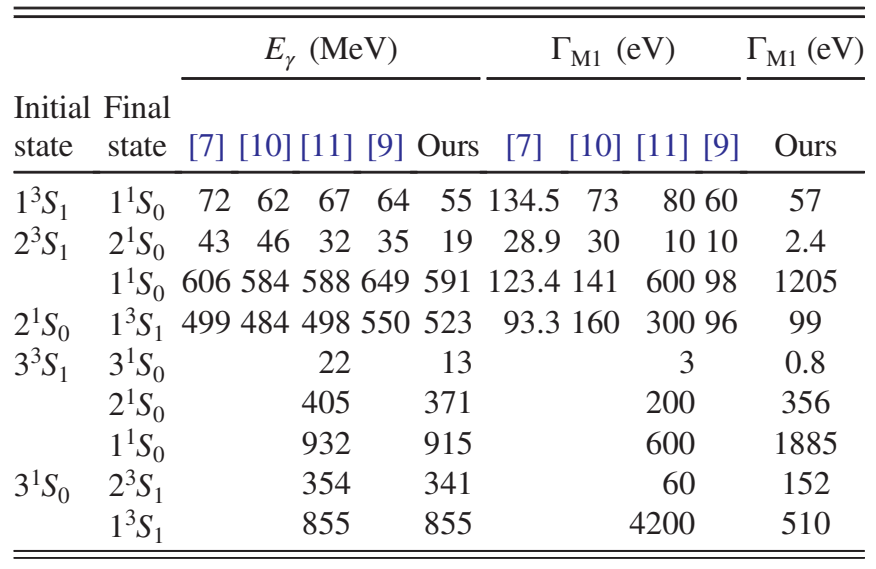


TABLE IV. Partial widths of the $M 1$ transitions for the higher $n S$-wave $(n=4,5,6) B_{c}$ states.

\begin{tabular}{|c|c|c|c|c|c|c|c|}
\hline $\begin{array}{l}\text { Initial } \\
\text { state }\end{array}$ & $\begin{array}{l}\text { Final } \\
\text { state }\end{array}$ & $\begin{array}{c}E_{\gamma} \\
(\mathrm{MeV})\end{array}$ & $\begin{array}{l}\Gamma_{\mathrm{EM}} \\
(\mathrm{eV})\end{array}$ & $\begin{array}{c}\text { Initial } \\
\text { state }\end{array}$ & $\begin{array}{l}\text { Final } \\
\text { state }\end{array}$ & $\begin{array}{c}E_{\gamma} \\
(\mathrm{MeV})\end{array}$ & $\begin{array}{l}\Gamma_{\mathrm{EM}} \\
(\mathrm{eV})\end{array}$ \\
\hline $4^{1} S_{0}$ & $\begin{array}{l}3^{3} S_{1} \\
2^{3} S_{1} \\
1^{3} S_{1}\end{array}$ & $\begin{array}{r}283 \\
622 \\
1116\end{array}$ & $\begin{array}{r}186 \\
579 \\
1122\end{array}$ & $4^{3} S_{1}$ & $\begin{array}{l}4^{1} S_{0} \\
3^{1} S_{0} \\
2^{1} S_{0} \\
{ }^{1} S_{0}\end{array}$ & $\begin{array}{r}10 \\
305 \\
648 \\
1171\end{array}$ & $\begin{array}{c}0.35 \\
252 \\
806 \\
2501\end{array}$ \\
\hline $5^{1} S_{0}$ & $\begin{array}{l}4{ }^{3} S_{1} \\
3^{3} S_{1} \\
2^{3} S_{1} \\
1^{3} S_{1}\end{array}$ & $\begin{array}{r}251 \\
533 \\
861 \\
1339\end{array}$ & $\begin{array}{r}209 \\
720 \\
1260 \\
1893\end{array}$ & $5^{3} S_{1}$ & $\begin{array}{l}5^{1} S_{0} \\
4^{1} S_{0} \\
3^{1} S_{0} \\
2^{1} S_{0} \\
{ }^{1} S_{0}\end{array}$ & $\begin{array}{r}8 \\
268 \\
553 \\
885 \\
1390\end{array}$ & $\begin{array}{c}0.18 \\
210 \\
675 \\
1316 \\
3107\end{array}$ \\
\hline $6^{1} S_{0}$ & $\begin{array}{l}5^{3} S_{1} \\
4^{3} S_{1} \\
3^{3} S_{1} \\
2^{3} S_{1} \\
1^{3} S_{1}\end{array}$ & $\begin{array}{r}230 \\
481 \\
755 \\
1073 \\
1536\end{array}$ & $\begin{array}{r}225 \\
849 \\
1613 \\
2203 \\
2822\end{array}$ & $6^{3} S_{1}$ & $\begin{array}{l}6^{1} S_{0} \\
5^{1} S_{0} \\
4^{1} S_{0} \\
3^{1} S_{0} \\
2^{1} S_{0} \\
1^{1} S_{0}\end{array}$ & $\begin{array}{r}8 \\
245 \\
498 \\
774 \\
1096 \\
1586\end{array}$ & $\begin{array}{c}0.18 \\
191 \\
643 \\
1239 \\
1917 \\
3772\end{array}$ \\
\hline
\end{tabular}

mesons. In this model, it assumes that the vacuum produces a quark-antiquark pair with the quantum number $0^{++}$and the heavy meson decay takes place via the rearrangement of the four quarks. The transition operator $\hat{T}$ in this model can be written as

$$
\begin{aligned}
\hat{T}= & -3 \gamma \sqrt{96 \pi} \sum_{m}\langle 1 m 1-m \mid 00\rangle \int d \mathbf{p}_{3} d \mathbf{p}_{4} \delta^{3}\left(\mathbf{p}_{3}+\mathbf{p}_{4}\right) \\
& \times \mathcal{Y}_{1}^{m}\left(\frac{\mathbf{p}_{3}-\mathbf{p}_{4}}{2}\right) \chi_{1-m}^{34} \phi_{0}^{34} \omega_{0}^{34} b_{3 i}^{\dagger}\left(\mathbf{p}_{3}\right) d_{4 j}^{\dagger}\left(\mathbf{p}_{4}\right)
\end{aligned}
$$

where $\gamma$ is a dimensionless constant that denotes the strength of the quark-antiquark pair creation with momentum $\mathbf{p}_{3}$ and $\mathbf{p}_{4}$ from vacuum; $b_{3 i}^{\dagger}\left(\mathbf{p}_{3}\right)$ and $d_{4 j}^{\dagger}\left(\mathbf{p}_{4}\right)$ are the creation operators for the quark and antiquark, respectively; the subscriptions, $i$ and $j$, are the $\mathrm{SU}(3)$-color indices of the created quark and antiquark; $\phi_{0}^{34}=(u \bar{u}+d \bar{d}+s \bar{s}) / \sqrt{3}$ and $\omega_{0}^{34}=\frac{1}{\sqrt{3}} \delta_{i j}$ correspond to flavor and color singlets, respectively; $\chi_{1,-m}^{34}$ is a spin triplet state; and

TABLE V. Partial widths of the $E 1$ dominant radiative transitions for the $1 P-, 1 D$-, and $1 F$-wave $B_{c}$ states. For comparison, the predictions from the relativistic quark model [10], relativized quark model [11], nonrelativistic constituent quark models [7,9] are listed

\begin{tabular}{|c|c|c|c|c|c|c|c|c|c|c|c|}
\hline \multirow[b]{2}{*}{ Initial state } & \multirow[b]{2}{*}{ Final state } & \multicolumn{5}{|c|}{$E_{\gamma}(\mathrm{MeV})$} & \multicolumn{4}{|c|}{$\Gamma_{\mathrm{E} 1}(\mathrm{keV})$} & \multirow{2}{*}{$\frac{\Gamma_{\mathrm{EM}}(\mathrm{keV})}{\text { Ours }}$} \\
\hline & & [7] & [10] & [11] & [9] & Ours & [7] & [10] & [11] & [9] & \\
\hline \multirow{10}{*}{$\begin{array}{l}1^{3} P_{2} \\
1 P_{1}^{\prime} \\
1 P_{1} \\
1^{3} P_{0} \\
1 P_{1}^{\prime} \\
1 P_{1} \\
1^{3} D_{3} \\
1 D_{2}^{\prime}\end{array}$} & \multirow{4}{*}{$1^{3} S_{1}$} & 397 & 416 & 416 & 426 & 445 & 112.6 & 122 & 83 & 102.9 & 87 \\
\hline & & 387 & 405 & 399 & 412 & 433 & 0.1 & 13.7 & 11 & 8.1 & 40 \\
\hline & & 382 & 389 & 391 & 400 & 416 & 99.5 & 87.1 & 60 & 77.8 & 70 \\
\hline & & 353 & 355 & 358 & 366 & 377 & 79.2 & 75.5 & 55 & 65.3 & 96 \\
\hline & \multirow[t]{2}{*}{$1^{1} S_{0}$} & 455 & 463 & 462 & 476 & 484 & 56.4 & 147 & 80 & 131.1 & 74 \\
\hline & & 450 & 447 & 454 & 464 & 468 & 0 & 18.4 & 13 & 11.6 & 35 \\
\hline & $1^{3} P_{2}$ & 258 & 312 & 272 & 264 & 239 & 98.7 & 149 & 78 & 76.9 & 67 \\
\hline & $1^{3} P_{2}$ & & 310 & 263 & 273 & 241 & & 12.6 & 8.8 & 6.8 & 8.3 \\
\hline & $1 P_{1}^{\prime}$ & & 321 & 280 & 287 & 253 & & 143 & 63 & 46.0 & 41 \\
\hline & $1 P_{1}$ & & 338 & 289 & 301 & 271 & & 7.1 & 7 & 25.0 & 0.39 \\
\hline \multirow[t]{3}{*}{$1 D_{2}$} & $1^{3} P_{2}$ & & 308 & 268 & 258 & 233 & & 23.6 & 9.6 & 12.2 & 8.7 \\
\hline & $1 P_{1}^{\prime}$ & & 319 & 285 & 272 & 246 & & 14.9 & 15 & 18.4 & 1.09 \\
\hline & $1 P_{1}$ & & 335 & 294 & 284 & 263 & & 139 & 64 & 44.6 & 44 \\
\hline \multirow[t]{4}{*}{$1^{3} D_{1}$} & $1^{3} P_{2}$ & 258 & 303 & 255 & 265 & 229 & 2.7 & 3.82 & 1.8 & 2.2 & 0.7 \\
\hline & $1 P_{1}^{\prime}$ & 268 & 315 & 273 & 279 & 242 & 0 & 7.81 & 4.4 & 3.3 & 12 \\
\hline & $1 P_{1}$ & 331 & 315 & 281 & 291 & 259 & 49.3 & 65.3 & 28 & 39.2 & 29 \\
\hline & $1^{3} P_{0}$ & 302 & 365 & 315 & 325 & 299 & 88.6 & 133 & 55 & 79.9 & 65 \\
\hline $1^{3} F_{4}$ & $1^{3} D_{3}$ & & & 222 & & 194 & & & 81 & & 69 \\
\hline \multirow[t]{3}{*}{$1 F_{3}^{\prime}$} & $1^{3} D_{3}$ & & & 227 & & 207 & & & 5.4 & & 4.76 \\
\hline & $1 D_{2}^{\prime}$ & & & 231 & & 205 & & & 82 & & 32 \\
\hline & $1 D_{2}$ & & & 236 & & 212 & & & 0.04 & & 0.04 \\
\hline \multirow[t]{3}{*}{$1 F_{3}$} & $1^{3} D_{3}$ & & & 218 & & 191 & & & 3.7 & & 4.91 \\
\hline & $1 D_{2}^{\prime}$ & & & 222 & & 189 & & & 0.5 & & 0.22 \\
\hline & $1 D_{2}$ & & & 226 & & 197 & & & 78 & & 29 \\
\hline \multirow[t]{4}{*}{$1^{3} F_{2}$} & $1^{3} D_{3}$ & & & 221 & & 202 & & & 0.4 & & 0.12 \\
\hline & $1 D_{2}^{\prime}$ & & & 224 & & 200 & & & 6.3 & & 5.72 \\
\hline & $1 D_{2}$ & & & 229 & & 208 & & & 6.5 & & 6.36 \\
\hline & $1^{3} D_{1}$ & & & 237 & & 212 & & & 75 & & 78 \\
\hline
\end{tabular}
in the table as well. 
TABLE VI. Partial widths of the $E 1$ dominant radiative transitions for the $2 D$-wave $B_{c}$ states.

\begin{tabular}{|c|c|c|c|c|c|c|c|}
\hline Initial state & Final state & $E_{\gamma}(\mathrm{MeV})$ & $\Gamma_{\mathrm{EM}}(\mathrm{keV})$ & Initial state & Final state & $E_{\gamma}(\mathrm{MeV})$ & $\Gamma_{\mathrm{EM}}(\mathrm{keV})$ \\
\hline \multirow{8}{*}{$2^{3} D_{1}$} & $1^{3} P_{2}$ & 528 & 8.13 & \multirow[t]{8}{*}{$2^{3} D_{3}$} & $1^{3} P_{2}$ & 540 & 32 \\
\hline & $1 P^{\prime}$ & 540 & 7.6 & & $1 P^{\prime}$ & 552 & 0.54 \\
\hline & $1 P$ & 557 & 12.5 & & $1 P$ & 568 & 1.23 \\
\hline & $1^{3} P_{0}$ & 596 & 41.8 & & $1^{3} P_{0}$ & 607 & 2.04 \\
\hline & $2^{3} P_{2}$ & 174 & 0.58 & & $2^{3} P_{2}$ & 186 & 54 \\
\hline & $2 P^{\prime}$ & 184 & 10.15 & & $2 P^{\prime}$ & 195 & 0.09 \\
\hline & $2 P$ & 199 & 20.88 & & $2 P$ & 211 & 0.23 \\
\hline & $2^{3} P_{0}$ & 225 & 46 & & $2^{3} P_{0}$ & 237 & 0.05 \\
\hline \multirow[t]{8}{*}{$2 D_{2}$} & $1^{3} P_{2}$ & 535 & 7.04 & \multirow[t]{8}{*}{$2 D_{2}^{\prime}$} & $1^{3} P_{2}$ & 539 & 7.28 \\
\hline & $1 P^{\prime}$ & 547 & 0.12 & & $1 P^{\prime}$ & 551 & 19 \\
\hline & $1 P$ & 564 & 22.6 & & $1 P$ & 567 & 1.48 \\
\hline & $1^{3} P_{0}$ & 602 & 0.29 & & $1^{3} P_{0}$ & 606 & 0.3 \\
\hline & $2^{3} P_{2}$ & 181 & 6.33 & & $2^{3} P_{2}$ & 185 & 6.71 \\
\hline & $2 P^{\prime}$ & 190 & 0.74 & & $2 P^{\prime}$ & 194 & 29 \\
\hline & $2 P$ & 206 & 34 & & $2 P$ & 210 & 0.24 \\
\hline & $2^{3} P_{0}$ & 232 & 0.04 & & $2^{3} P_{0}$ & 236 & 0.05 \\
\hline
\end{tabular}

TABLE VII. Partial widths of the $E 1$ dominant radiative transitions for the $2 S$-, $2 P$-wave $B_{c}$ states. For comparison, the predictions from the relativistic quark model [10], relativized quark model [11], nonrelativistic constituent quark models [7,9] are listed in the table as well.

\begin{tabular}{|c|c|c|c|c|c|c|c|c|c|c|c|}
\hline \multirow[b]{2}{*}{ Initial state } & \multirow[b]{2}{*}{ Final state } & \multicolumn{5}{|c|}{$E_{\gamma}(\mathrm{MeV})$} & \multicolumn{4}{|c|}{$\Gamma_{\mathrm{E} 1}(\mathrm{keV})$} & \multirow{2}{*}{$\frac{\Gamma_{\mathrm{EM}}(\mathrm{keV})}{\text { Ours }}$} \\
\hline & & [7] & [10] & [11] & [9] & Ours & [7] & {$[10]$} & [11] & [9] & \\
\hline \multirow{4}{*}{$2^{3} S_{1}$} & $1^{3} P_{2}$ & 151 & 118 & 118 & 159 & 102 & 17.7 & 7.59 & 5.7 & 14.8 & 6.98 \\
\hline & $1 P_{1}^{\prime}$ & 161 & 130 & 136 & 173 & 115 & 0 & 0.74 & 0.7 & 1.0 & 1.56 \\
\hline & $1 P_{1}$ & 167 & 146 & 144 & 185 & 133 & 14.5 & 7.65 & 4.7 & 12.8 & 4.62 \\
\hline & $1^{3} P_{0}$ & 196 & 181 & 179 & 219 & 174 & 7.8 & 5.53 & 2.9 & 7.7 & 3.48 \\
\hline \multirow[t]{2}{*}{$2^{1} S_{0}$} & $1 P_{1}^{\prime}$ & 119 & 84 & 104 & 138 & 96 & 5.2 & 4.40 & 6.1 & 15.9 & 6.38 \\
\hline & $1 P_{1}$ & 125 & 101 & 113 & 150 & 114 & 0 & 1.05 & 1.3 & 1.9 & 5.33 \\
\hline \multirow[t]{6}{*}{$2^{3} P_{2}$} & $1^{3} D_{3}$ & 142 & 75 & 118 & 127 & 129 & 17.8 & 2.08 & 6.8 & 10.9 & 14 \\
\hline & $1 D_{2}^{\prime}$ & & 77 & 122 & 118 & 127 & & 0.139 & 0.6 & 0.5 & 0.93 \\
\hline & $1 D_{2}$ & & 79 & 127 & 133 & 135 & & 0.285 & 0.7 & 1.5 & 1.1 \\
\hline & $1^{3} D_{1}$ & 142 & 84 & 135 & 126 & 139 & 0.2 & 0.035 & 0.1 & 0.1 & 0.13 \\
\hline & $2^{3} S_{1}$ & 249 & 270 & 272 & 232 & 265 & 73.8 & 75.3 & 55 & 49.4 & 50 \\
\hline & $1^{3} S_{1}$ & 770 & & 778 & 817 & 785 & 25.8 & & 14 & 25.8 & 52 \\
\hline \multirow[t]{7}{*}{$2 P_{1}^{\prime}$} & $1 D_{2}^{\prime}$ & & 66 & 113 & 108 & 117 & & 1.49 & 5.5 & 3.5 & 1.05 \\
\hline & $1 D_{2}$ & & 68 & 123 & 123 & 125 & & 0.172 & 1.3 & 2.5 & 0.03 \\
\hline & $1^{3} D_{1}$ & 131 & 73 & 121 & 116 & 129 & 0.4 & 0.07 & 0.2 & 0.3 & 1.27 \\
\hline & $2^{3} S_{1}$ & 239 & 259 & 258 & 222 & 255 & 5.4 & 10.4 & 5.5 & 5.9 & 25 \\
\hline & $1^{3} S_{1}$ & 760 & & 769 & 807 & 777 & 2.1 & & 0.6 & 2.5 & 26 \\
\hline & $2^{1} S_{0}$ & & 303 & 289 & 257 & 274 & & 90.5 & 52 & 58.0 & 36 \\
\hline & $1^{1} S_{0}$ & & & 825 & 871 & 825 & & & 19 & 131.1 & 44 \\
\hline \multirow[t]{7}{*}{$2 P_{1}$} & $1 D_{2}^{\prime}$ & & 47 & 108 & 97 & 101 & & 0.023 & 0.8 & 1.2 & 0.006 \\
\hline & $1 D_{2}$ & & 49 & 103 & 112 & 109 & & 0.517 & 3.6 & 3.9 & 0.84 \\
\hline & $1^{3} D_{1}$ & 125 & 54 & 116 & 105 & 113 & 0.3 & 0.204 & 1.6 & 1.6 & 1.45 \\
\hline & $2^{3} S_{1}$ & 232 & 241 & 253 & 211 & 240 & 54.3 & 45.3 & 45 & 32.1 & 34 \\
\hline & $1^{3} S_{1}$ & 754 & & 761 & 796 & 762 & 22.1 & & 5.4 & 15.3 & 40 \\
\hline & $2^{1} S_{0}$ & & 285 & 284 & 246 & 258 & & 13.8 & 5.7 & 8.1 & 19 \\
\hline & $1^{1} S_{0}$ & & & 820 & 860 & 811 & & & 2.1 & 3.1 & 25 \\
\hline \multirow[t]{3}{*}{$2^{3} P_{0}$} & $1^{3} D_{1}$ & 98 & 19 & 93 & 80 & 86 & 6.9 & 0.041 & 4.2 & 3.2 & 5.6 \\
\hline & $2^{3} S_{1}$ & 205 & 207 & 231 & 186 & 214 & 41.2 & 34 & 42 & 25.5 & 53 \\
\hline & $1^{3} S_{1}$ & 729 & & 741 & 771 & 738 & 21.9 & & 1 & 16.1 & 41 \\
\hline
\end{tabular}


TABLE VIII. Partial widths of the $E 1$ dominant radiative transitions for the $3 S$-, $4 S$-, $3 P$-wave $B_{c}$ states.

\begin{tabular}{|c|c|c|c|c|c|c|c|}
\hline Initial state & Final state & $E_{\gamma}(\mathrm{MeV})$ & $\Gamma_{\mathrm{EM}}(\mathrm{keV})$ & Initial state & Final state & $E_{\gamma}(\mathrm{MeV})$ & $\Gamma_{\mathrm{EM}}(\mathrm{keV})$ \\
\hline $3^{1} S_{0}$ & $\begin{array}{l}2 P^{\prime} \\
2 P \\
1 P^{\prime} \\
1 P\end{array}$ & $\begin{array}{r}88 \\
104 \\
450 \\
467\end{array}$ & $\begin{array}{r}11.13 \\
10.93 \\
1.74 \\
1.25\end{array}$ & $3^{3} S_{1}$ & $\begin{array}{c}2^{3} P_{2} \\
2 P^{\prime} \\
2 P \\
2^{3} P_{0} \\
1^{3} P_{2} \\
1 P^{\prime} \\
1 P \\
1^{3} P_{0}\end{array}$ & $\begin{array}{r}91 \\
101 \\
117 \\
144 \\
450 \\
462 \\
479 \\
518\end{array}$ & $\begin{array}{l}11.89 \\
2.92 \\
7.2 \\
5 \\
1.58 \\
0.7 \\
1.72 \\
1.73\end{array}$ \\
\hline $4^{1} S_{0}$ & $\begin{array}{l}1 P^{\prime} \\
1 P \\
2 P^{\prime} \\
2 P \\
3 P^{\prime} \\
3 P\end{array}$ & $\begin{array}{r}727 \\
743 \\
380 \\
395 \\
82 \\
98\end{array}$ & $\begin{array}{l}1.93 \\
1.7 \\
6.31 \\
5.14 \\
13 \\
17\end{array}$ & $4^{3} S_{1}$ & $\begin{array}{c}1^{3} P_{2} \\
1 P^{\prime} \\
1 P \\
1^{3} P_{0} \\
2^{3} P_{2} \\
2 P^{\prime} \\
2 P \\
2^{3} P_{0} \\
3^{3} P_{2} \\
3 P^{\prime} \\
3 P \\
3^{3} P_{0}\end{array}$ & $\begin{array}{r}724 \\
736 \\
752 \\
790 \\
380 \\
389 \\
405 \\
430 \\
86 \\
91 \\
108 \\
129\end{array}$ & $\begin{array}{c}1.88 \\
0.82 \\
1.37 \\
1.3 \\
5.78 \\
1.96 \\
4.04 \\
3.28 \\
16 \\
4.06 \\
8.71 \\
6.12\end{array}$ \\
\hline $3^{3} P_{0}$ & $\begin{array}{l}2^{3} D_{1} \\
1^{3} D_{1} \\
3^{3} S_{1} \\
2^{3} S_{1} \\
1^{3} S_{1}\end{array}$ & $\begin{array}{r}84 \\
389 \\
166 \\
511 \\
1013\end{array}$ & $\begin{array}{l}10.93 \\
1.84 \\
45 \\
36 \\
30\end{array}$ & $3^{3} P_{2}$ & $\begin{array}{c}2^{3} D_{3} \\
2 D^{\prime} \\
2 D \\
2^{3} D_{1} \\
1^{3} D_{3} \\
1 D^{\prime} \\
1 D \\
1^{3} D_{1} \\
3^{3} S_{1} \\
2^{3} S_{1} \\
1^{3} S_{1}\end{array}$ & $\begin{array}{r}115 \\
116 \\
120 \\
127 \\
421 \\
419 \\
427 \\
431 \\
209 \\
552 \\
1051\end{array}$ & $\begin{array}{l}22 \\
1.57 \\
1.72 \\
0.23 \\
9.07 \\
1.06 \\
1.16 \\
0.94 \\
43 \\
39 \\
42\end{array}$ \\
\hline $3 P_{1}$ & $\begin{array}{c}2 D^{\prime} \\
2 D \\
2^{3} D_{1} \\
1 D^{\prime} \\
1 D \\
1^{3} D_{1} \\
3^{3} S_{1} \\
2^{3} S_{1} \\
1^{3} S_{1} \\
3^{1} S_{0} \\
2^{1} S_{0} \\
1^{1} S_{0} \\
\end{array}$ & $\begin{array}{r}93 \\
97 \\
104 \\
398 \\
405 \\
409 \\
187 \\
531 \\
1031 \\
199 \\
548 \\
1078 \\
\end{array}$ & $\begin{array}{l}0.003 \\
1.3 \\
2.39 \\
0.74 \\
0.31 \\
0.67 \\
26 \\
27 \\
30 \\
18 \\
19 \\
23 \\
\end{array}$ & $3 P_{1}^{\prime}$ & $\begin{array}{c}2 D^{\prime} \\
2 D \\
2^{3} D_{1} \\
1 D^{\prime} \\
1 D \\
1^{3} D_{1} \\
3^{3} S_{1} \\
2^{3} S_{1} \\
1^{3} S_{1} \\
3^{1} S_{0} \\
2^{1} S_{0} \\
1^{1} S_{0} \\
\end{array}$ & $\begin{array}{r}110 \\
114 \\
121 \\
414 \\
421 \\
425 \\
203 \\
546 \\
1046 \\
216 \\
564 \\
1093 \\
\end{array}$ & $\begin{array}{l}1.9 \\
0.05 \\
2.47 \\
0.19 \\
0.93 \\
0.61 \\
25 \\
22 \\
24 \\
30 \\
28 \\
32 \\
\end{array}$ \\
\hline
\end{tabular}

$\mathcal{Y}_{\ell m}(\mathbf{k}) \equiv|\mathbf{k}|^{\ell} Y_{\ell m}\left(\theta_{\mathbf{k}}, \phi_{\mathbf{k}}\right)$ is the $\ell$-th solid harmonic polynomial. The factor $(-3)$ is introduced for convenience, which will cancel the color factor.

For an OZI-allowed two-body strong decay process $A \rightarrow B+C$, the helicity amplitude $\mathcal{M}^{M_{J_{A}} M_{J_{B}} M_{J_{C}}}(\mathbf{P})$ can be derived as follows:

$$
\langle B C|T| A\rangle=\delta\left(\mathbf{P}_{A}-\mathbf{P}_{B}-\mathbf{P}_{C}\right) \mathcal{M}^{M_{J_{A}} M_{J_{B}} M_{J_{C}}}(\mathbf{P})
$$

Using the Jacob-Wick formula [79], one can convert the helicity amplitudes $\mathcal{M}^{M_{J_{A}} M_{J_{B}} M_{J_{C}}}(\mathbf{P})$ to the partial wave amplitudes $\mathcal{M}^{J L}$ via

$$
\begin{aligned}
\mathcal{M}^{J L}(A \rightarrow B C)= & \frac{\sqrt{4 \pi(2 L+1)}}{2 J_{A}+1} \sum_{M_{J_{B}}, M_{J_{C}}}\left\langle L 0 J M_{J_{A}} \mid J_{A} M_{J_{A}}\right\rangle \\
& \times\left\langle J_{B} M_{J_{B}} J_{C} M_{J_{C}} \mid J M_{J_{A}}\right\rangle \mathcal{M}^{M_{J_{A}} M_{J_{B}} M_{J_{C}}(\mathbf{P}) .}
\end{aligned}
$$


TABLE IX. The masses $(\mathrm{MeV})$ of the final hadrons appearing in the strong decay processes of the $B_{c}$ states. The masses are taken from the Particle Data Group [83] if there are experimental data; otherwise we take the quark model predictions in Refs. [81,82].

\begin{tabular}{lcccccc}
\hline \hline State & $1{ }^{1} S_{0}$ & $1{ }^{3} S_{1}$ & $1{ }^{3} P_{0}$ & $1 P_{1}$ & $1 P_{1}^{\prime}$ & $1^{3} P_{2}$ \\
\hline$B$ & 5279 & 5325 & 5683 & 5729 & 5754 & 5768 \\
$B_{s}$ & 5367 & 5415 & 5756 & 5801 & 5836 & 5851 \\
$D$ & 1870 & 2010 & 2252 & 2402 & 2417 & 2466 \\
$D_{s}$ & 1968 & 2112 & 2344 & 2488 & 2510 & 2559 \\
\hline \hline
\end{tabular}

In the above equations, $\left(J_{A}, J_{B}\right.$ and $\left.J_{C}\right),\left(L_{A}, L_{B}\right.$ and $\left.L_{C}\right)$, and $\left(S_{A}, S_{B}\right.$ and $\left.S_{C}\right)$ are the quantum numbers of the total angular momenta, orbital angular momenta, and total spin for hadrons $A, B, C$, respectively; $M_{J_{A}}=M_{J_{B}}+M_{J_{C}}$, $\mathbf{J} \equiv \mathbf{J}_{B}+\mathbf{J}_{C}$, and $\mathbf{J}_{A} \equiv \mathbf{J}_{B}+\mathbf{J}_{C}+\mathbf{L}$. In the c.m. frame of hadron $A$, the momenta $\mathbf{P}_{B}$ and $\mathbf{P}_{C}$ of mesons $B$ and $C$ satisfy $\mathbf{P}_{B}=-\mathbf{P}_{C} \equiv \mathbf{P}$.

Then the strong decay partial width for a given decay mode of $A \rightarrow B+C$ is given by

$$
\Gamma=2 \pi|\mathbf{P}| \frac{E_{B} E_{C}}{M_{A}} \sum_{J L}\left|\mathcal{M}^{J L}\right|^{2},
$$

where $M_{A}$ is the mass of the initial hadron $A$, while $E_{B}$ and $E_{C}$ stand for the energies of final hadrons $B$ and $C$, respectively. The details of the ${ }^{3} P_{0}$ model can be found in our recent paper [80].

In the calculations, the wave functions of the initial $B_{c}$ states are adopted from our quark model predictions. Furthermore, we need the wave functions of the final hadrons, i.e., the $B^{(*)}, B_{s}^{(*)}, D^{(*)}, D_{s}^{(*)}$ mesons and some of their excitations, which are adopted from the quark model predictions of Refs. [81,82].

In this work, for the masses of the light constituent $u, d$, and $s$ quarks, we set $m_{u}=m_{d}=0.33 \mathrm{GeV}, m_{s}=0.45 \mathrm{GeV}$; while for the heavy $b$ and $c$ quarks, their masses are taken to be $m_{b}=4.852 \mathrm{GeV}$ and $m_{c}=1.483 \mathrm{GeV}$ as the determinations in the calculations of the $B_{c}$ mass spectrum. The masses of the final hadron states in the decay processes are adopted from the Particle Data Group [83] if there are measured values; otherwise we take the quark model predictions of Refs. [81,82] (see Table IX). There is no experimental data which can be used to determine the quark pair creation strength; thus, in this work we adopt a typical value $\gamma=0.4$ that gives a reasonably accurate description of the overall scale of decay widths of both light and heavy mesons [66,84-88]. The strong decay properties for the bottom-charmed states are presented in Table X-XV.

\section{DISCUSSION}

\section{A. $S$-wave states}

Recently, signals of two excited $\bar{b} c$ states, $B_{c}(2 S)$ and $B_{c}^{*}(2 S)$, were observed in the $B_{c}^{+} \pi^{+} \pi^{-}$invariant mass spectrum by the CMS Collaboration at LHC. These two states are well resolved from each other and are observed with a significance exceeding five standard deviations. The mass of $B_{c}(2 S)$ meson is measured to be $6871 \pm 2.8 \mathrm{MeV}$. Furthermore, a more precise mass of $B_{c}(2 S), M\left(B_{c}^{+}\right)=6871.1 \pm 0.5 \mathrm{MeV}$, is measured by the CMS Collaboration as well. Combining these newest measurements, we predict that the mass of $B_{c}(2 S)$ might be $\sim 6890 \mathrm{MeV}$, and the mass hyperfine splitting between $B_{c}^{*}(2 S)$ and $B_{c}(2 S)$,

$$
\Delta m(2 S) \simeq 20 \mathrm{MeV},
$$

is slightly smaller than $30-45 \mathrm{MeV}$, predicted in previous works (see Table I). The predicted masses for the other higher $S$-wave states compared with other works are also given in Table I. Obvious differences can be found in various theoretical predictions.

The $M 1$ transitions of the low-lying $S$-wave states $B_{c}^{*}(2 S)$ and $B_{c}^{(*)}(1 S)$ were often discussed in the literature for these transitions which might be used to establish them in experiments. In this work we also calculate their M1 transitions. Our results compared with some other predictions are listed Table III. Obvious model dependence can be seen in various calculations. Our predicted partial width,

$$
\Gamma\left[B_{c}^{*}(2 S) \rightarrow B_{c} \gamma\right] \simeq 1.2 \mathrm{keV},
$$

for the $M 1$ transition $B_{c}^{*}(2 S) \rightarrow B_{c} \gamma$ is about an order of magnitude larger than that predicted in Refs. [7,9,10], and about a factor 2 larger than the value predicted within the GI model [11]. Combining our calculations of the EM transitions $B_{c}^{*}(2 S) \rightarrow 1 P \gamma$ and the strong transitions $B_{c}^{*}(2 S) \rightarrow B_{c}^{*} \pi \pi$ predicted in [11], the total decay width of $B_{c}^{*}(2 S)$ meson is estimated to be $\Gamma_{\text {total }} \sim 75 \mathrm{keV}$; then the branching fraction for $M 1$ transition $B_{c}^{*}(2 S) \rightarrow B_{c} \gamma$ is predicted to be

$$
\operatorname{Br}\left[B_{c}^{*}(2 S) \rightarrow B_{c} \gamma\right] \sim 2 \%
$$

The fairly large branching fraction may give a good opportunity for us to observe the $B_{c}^{*}(2 S)$ via the $M 1$ transition $B_{c}^{*}(2 S) \rightarrow B_{c} \gamma$. This process may be used to determined the mass of $B_{c}^{*}(2 S)$ in future experiments.

The masses of $3 S$-wave states $B_{c}\left(3^{1} S_{0}\right)$ and $B_{c}\left(3^{3} S_{1}\right)$ are predicted to be $\sim 7.24 \mathrm{GeV}$ and $\sim 7.25 \mathrm{GeV}$, respectively, which are just above the $D B^{*}$ threshold. Their radiative and strong decay properties are estimated in this work. The results for the $M 1$ transitions, $E 1$ dominant transitions, and strong decays of the $3 S$-wave states are given in Tables III, VI, and X, respectively. There are only a few works about the radiative and strong decay properties of the $3 S$-wave states $[11,18,59,60]$. The $M 1$ transitions of the $3 S$-wave states roughly agree with the predictions in Ref. [11], except that our predicted partial width $\Gamma\left[3{ }^{3} S_{1} \rightarrow{ }^{1} S_{0}+\gamma\right] \simeq 510 \mathrm{eV}$ for the $M 1$ transition 
TABLE X. Strong decay properties for the $4 S$-, $5 S$-wave $B_{c}$ states. $\Gamma$ th and $B_{r}$ stand for the partial widths and branching ratios of the strong decay processes, respectively.

\begin{tabular}{|c|c|c|c|c|c|c|c|}
\hline State & Decay mode & $\Gamma_{t h}(\mathrm{MeV})$ & $B_{r}(\%)$ & State & Decay mode & $\Gamma_{t h}(\mathrm{MeV})$ & $B_{r}(\%)$ \\
\hline \multirow{3}{*}{$3^{1} S_{0}(7239)$} & $B^{*} D$ & 161 & 100 & $3^{3} S_{1}(7252)$ & $B D$ & 28 & 21 \\
\hline & & & & & $B^{*} D$ & 105 & 79 \\
\hline & Total & 161 & 100 & & Total & 133 & 100 \\
\hline \multirow[t]{9}{*}{$4^{1} S_{0}(7540)$} & $B^{*} D$ & 0.14 & 0.1 & $4^{3} S_{1}(7550)$ & $B D$ & 4.53 & 2.7 \\
\hline & $B D^{*}$ & 34.9 & 18.3 & & $B^{*} D$ & 0.41 & 0.2 \\
\hline & $B^{*} D^{*}$ & 104 & 54 & & $B D^{*}$ & 17.0 & 10 \\
\hline & $B_{s}^{* 0} D_{s}^{+}$ & 6.7 & 3.5 & & $B^{*} D^{*}$ & 112 & 66 \\
\hline & $B_{s}^{0} D_{s}^{*+}$ & 5.8 & 3.1 & & $B_{s}^{0} D_{s}^{+}$ & 2.81 & 1.6 \\
\hline & $B_{s}^{* 0} D_{s}^{*+}$ & 15.5 & 8.1 & & $B_{s}^{* 0} D_{s}^{+}$ & 5.29 & 3.1 \\
\hline & $B D\left(1^{3} P_{0}\right)$ & 24 & 12.6 & & $B_{s}^{0} D_{s}^{*+}$ & 1.83 & 1.1 \\
\hline & & & & & $B_{s}^{* 0} D_{s}^{*+}$ & 26.9 & 16 \\
\hline & Total & 191 & 100 & & Total & 171 & 100 \\
\hline \multirow[t]{26}{*}{$5^{1} S_{0}(7805)$} & $B^{*} D$ & 24.5 & 5.9 & $5^{3} S_{1}(7813)$ & $B D$ & 15.81 & 3.9 \\
\hline & $B D^{*}$ & 1.5 & 0.4 & & $B^{*} D$ & 20.18 & 5 \\
\hline & $B^{*} D^{*}$ & 2.28 & 0.6 & & $B D^{*}$ & 2.65 & 0.7 \\
\hline & $B_{s}^{* 0} D_{s}^{+}$ & 1.62 & 0.4 & & $B^{*} D^{*}$ & 0.19 & 0.05 \\
\hline & $B_{s}^{0} D_{s}^{*+}$ & 4.65 & 1.1 & & $B_{s}^{0} D_{s}^{+}$ & 0.02 & 0.005 \\
\hline & $B_{s}^{* 0} D_{s}^{*+}$ & 5.75 & 1.4 & & $B_{s}^{* 0} D_{s}^{+}$ & 0.62 & 0.2 \\
\hline & $B\left(1^{3} P_{0}\right) D$ & 18.6 & 4.5 & & $B_{s}^{0} D_{s}^{*+}$ & 3.02 & 0.8 \\
\hline & $B\left(1^{3} P_{2}\right) D$ & 27.6 & 6.7 & & $B_{s}^{* 0} D_{s}^{*+}$ & 8.09 & 2 \\
\hline & $B\left(1 P^{\prime}\right) D^{*}$ & 82 & 19.9 & & $B\left(1 P^{\prime}\right) D$ & 18.96 & 4.7 \\
\hline & $B(1 P) D^{*}$ & 6.2 & 1.5 & & $B(1 P) D$ & 13.34 & 3.3 \\
\hline & $B\left(1^{3} P_{2}\right) D^{*}$ & 56.5 & 13.7 & & $B\left(1^{3} P_{2}\right) D$ & 16.1 & 4 \\
\hline & $B D\left(1^{3} P_{0}\right)$ & 23.5 & 5.7 & & $B\left(1^{3} P_{0}\right) D^{*}$ & 0.04 & 0.01 \\
\hline & $B D\left(1^{3} P_{2}\right)$ & 48.2 & 11.7 & & $B\left(1 P^{\prime}\right) D^{*}$ & 53.93 & 13.4 \\
\hline & $B^{*} D\left(1 P^{\prime}\right)$ & 70.9 & 17.2 & & $B(1 P) D^{*}$ & 5.19 & 1.3 \\
\hline & $B^{*} D(1 P)$ & 12.3 & 3.0 & & $B\left(1^{3} P_{2}\right) D^{*}$ & 96 & 24 \\
\hline & $B^{*} D\left(1^{3} P_{2}\right)$ & 25.7 & 6.2 & & $B D\left(1 P^{\prime}\right)$ & 0.89 & 0.2 \\
\hline & $B_{S}\left(1^{3} P_{0}\right) D_{S}$ & 0.17 & 0.04 & & $B D(1 P)$ & 0.63 & 0.2 \\
\hline & $B_{S} D_{S}\left(1^{3} P_{0}\right)$ & 0.56 & 0.14 & & $B D\left(1^{3} P_{2}\right)$ & 17.34 & 4.3 \\
\hline & & & & & $B^{*} D\left(1^{3} P_{0}\right)$ & 18.32 & 4.6 \\
\hline & & & & & $B^{*} D\left(1 P^{\prime}\right)$ & 32.9 & 8.2 \\
\hline & & & & & $B^{*} D(1 P)$ & 6.8 & 1.7 \\
\hline & & & & & $B^{*} D\left(1^{3} P_{2}\right)$ & 69.87 & 17 \\
\hline & & & & & $B_{S}\left(1 P^{\prime}\right) D_{S}$ & 0.36 & 0.09 \\
\hline & & & & & $B_{S}(1 P) D_{S}$ & 0.01 & 0.002 \\
\hline & & & & & $B_{S}^{*} D_{S}\left(1^{3} P_{0}\right)$ & 0.3 & 0.07 \\
\hline & Total & 413 & 100 & & Total & 401 & 100 \\
\hline
\end{tabular}

$3^{3} S_{1} \rightarrow{ }^{1} S_{0}+\gamma$ is about an order of magnitude smaller than that in Ref. [11]. The strong decay widths of $B_{c}\left(3{ }^{1} S_{0}\right)$ and $B_{c}\left(3^{3} S_{1}\right)$ predicted by us are comparable with those predicted in recent works $[18,59]$. Both $B_{c}\left(3^{1} S_{0}\right)$ and $B_{c}\left(3^{3} S_{1}\right)$ might be broad states with a width of $\sim 100 \mathrm{MeV}$. The $B_{c}\left(3^{1} S_{0}\right)$ dominantly decay into $D B^{*}$ channel, while $B_{c}\left(3^{3} S_{1}\right)$ dominantly decay into both $D B$ and $D B^{*}$ channels. The production rates of the $3 S$-wave $B_{c}$ states in $p p$ collisions at the LHC may be comparable with those of the $2 S$-wave $B_{c}$ states [18]; thus, the $3 S$-wave $B_{c}$ states may have large potentials to be established in the $D B^{*}$ final states.
The higher $S$-wave states $B_{c}\left(n^{1} S_{0}\right)$ and $B_{c}\left(n^{3} S_{1}\right)(n \geq 4)$ are far from the $D B$ threshold, thus many OZI-allowed twobody strong decay channels are open. There are few discussions of the decay properties of the higher mass $S$-wave states in the literature. To know some decay properties of these higher $S$-wave states, in this work we give our predictions of the $M 1$ transitions and strong decays of $B_{c}(n S)(n=4,5,6)$, which are listed in Tables IV and X, respectively. It is found that these higher mass $S$-wave states are broad states with a width of $\sim 100-400 \mathrm{MeV}$. Combining $M 1$ transitions of higher $S$-wave states with their strong decays, we find that the branching fractions of 
TABLE XI. Strong decay properties for the $6 S$-wave $B_{c}$ states.

\begin{tabular}{|c|c|c|c|c|c|c|c|}
\hline State & Decay mode & $\Gamma_{t h}(\mathrm{MeV})$ & $B_{r}(\%)$ & State & Decay mode & $\Gamma_{t h}(\mathrm{MeV})$ & $B_{r}(\%)$ \\
\hline \multirow[t]{37}{*}{$6^{1} S_{0}(8046)$} & $B^{*} D$ & 44.4 & 12 & $6^{3} S_{1}(8054)$ & $B D$ & 17.6 & 4.7 \\
\hline & $B D^{*}$ & 24.3 & 6.7 & & $B^{*} D$ & 31 & 8.3 \\
\hline & $B^{*} D^{*}$ & 24.3 & 6.7 & & $B D^{*}$ & 19.1 & 5.1 \\
\hline & $B_{s}^{* 0} D_{s}^{+}$ & 1.11 & 0.3 & & $B^{*} D^{*}$ & 37.9 & 10.2 \\
\hline & $B_{s}^{0} D_{s}^{*+}$ & 0.38 & 0.11 & & $B_{s}^{0} D_{s}^{+}$ & 1.78 & 0.5 \\
\hline & $B_{s}^{* 0} D_{s}^{*+}$ & 3.33 & 0.9 & & $B_{s}^{* 0} D_{s}^{+}$ & 1.22 & 0.3 \\
\hline & $B\left(1^{3} P_{0}\right) D$ & 11.3 & 3.1 & & $B_{s}^{0} D_{s}^{*+}$ & 0.09 & 0.02 \\
\hline & $B\left(1^{3} P_{2}\right) D$ & 4.85 & 1.3 & & $B_{s}^{* 0} D_{s}^{*+}$ & 2.96 & 0.8 \\
\hline & $B\left(1 P^{\prime}\right) D^{*}$ & 28.3 & 7.8 & & $B\left(1 P^{\prime}\right) D$ & 0.25 & 0.07 \\
\hline & $B(1 P) D^{*}$ & 24.7 & 6.8 & & $B(1 P) D$ & 11.1 & 3 \\
\hline & $B\left(1^{3} P_{2}\right) D^{*}$ & 20.6 & 5.7 & & $B\left(1^{3} P_{2}\right) D$ & 1.09 & 0.3 \\
\hline & $B D\left(1^{3} P_{0}\right)$ & 13.2 & 3.6 & & $B\left(1^{3} P_{0}\right) D^{*}$ & 10.9 & 3 \\
\hline & $B D\left(1^{3} P_{2}\right)$ & 28.9 & 8 & & $B\left(1 P^{\prime}\right) D^{*}$ & 21.2 & 5.7 \\
\hline & $B^{*} D\left(1 P^{\prime}\right)$ & 46.8 & 13 & & $B(1 P) D^{*}$ & 17 & 4.6 \\
\hline & $B^{*} D(1 P)$ & 41.4 & 11.4 & & $B\left(1^{3} P_{2}\right) D^{*}$ & 34 & 9.1 \\
\hline & $B^{*} D\left(1^{3} P_{2}\right)$ & 23.5 & 6.5 & & $B D\left(1 P^{\prime}\right)$ & 9.37 & 2.5 \\
\hline & $B_{s}\left(1^{3} P_{0}\right) D_{s}$ & 5.5 & 1.5 & & $B D(1 P)$ & 16.6 & 4.4 \\
\hline & $B_{s}\left(1^{3} P_{2}\right) D_{s}$ & 0.17 & 0.05 & & $B D\left(1^{3} P_{2}\right)$ & 14.1 & 3.8 \\
\hline & $B_{s}\left(1 P^{\prime}\right) D_{s}^{*}$ & 0.88 & 0.24 & & $B^{*} D\left(1^{3} P_{0}\right)$ & 12.9 & 3.5 \\
\hline & $B_{s}(1 P) D_{s}^{*}$ & 0.03 & 0.01 & & $B^{*} D\left(1 P^{\prime}\right)$ & 30.5 & 8.2 \\
\hline & $B_{s}\left(1^{3} P_{2}\right) D_{s}^{*}$ & 0.02 & 0.01 & & $B^{*} D(1 P)$ & 27.9 & 7.5 \\
\hline & $B_{s} D_{s}\left(1^{3} P_{0}\right)$ & 6.62 & 1.8 & & $B^{*} D\left(1^{3} P_{2}\right)$ & 39.9 & 10.7 \\
\hline & $B_{s} D_{s}\left(1^{3} P_{2}\right)$ & 2.47 & 0.68 & & $B_{s}\left(1 P^{\prime}\right) D_{s}$ & 0.61 & 0.2 \\
\hline & $B_{s}^{*} D_{s}\left(1 P^{\prime}\right)$ & 4.14 & 1.1 & & $B_{s}(1 P) D_{s}$ & 3.06 & 0.8 \\
\hline & $B_{s}^{*} D_{s}(1 P)$ & 0.23 & 0.06 & & $B_{s}\left(1^{3} P_{2}\right) D_{s}$ & 0.24 & 0.1 \\
\hline & $B_{s}^{*} D_{s}\left(1^{3} P_{2}\right)$ & 0.18 & 0.05 & & $B_{s}\left(1^{3} P_{0}\right) D_{s}^{*}$ & 0.001 & 0.0003 \\
\hline & & & & & $B_{s}\left(1 P^{\prime}\right) D_{s}^{*}$ & 1.13 & 0.3 \\
\hline & & & & & $B_{s}(1 P) D_{s}^{*}$ & 0.005 & 0.001 \\
\hline & & & & & $B_{s}\left(1^{3} P_{2}\right) D_{s}^{*}$ & 0.48 & 0.1 \\
\hline & & & & & $B_{s} D_{s}\left(1 P^{\prime}\right)$ & 0.56 & 0.2 \\
\hline & & & & & $B_{s} D_{s}(1 P)$ & 0.04 & 0.01 \\
\hline & & & & & $B_{s} D_{s}\left(1^{3} P_{2}\right)$ & 1.35 & 0.4 \\
\hline & & & & & $B_{s}^{*} D_{s}\left(1^{3} P_{0}\right)$ & 3.3 & 0.9 \\
\hline & & & & & $B_{s}^{*} D_{s}\left(1 P^{\prime}\right)$ & 3.06 & 0.8 \\
\hline & & & & & $B_{s}^{*} D_{s}(1 P)$ & 0.21 & 0.1 \\
\hline & & & & & $B_{s}^{*} D_{s}\left(1^{3} P_{2}\right)$ & 0.03 & 0.008 \\
\hline & Total & 361 & 100 & & Total & 372 & 100 \\
\hline
\end{tabular}

the $M 1$ transitions $B_{c}(n S) \rightarrow B_{c}(1 S)+\gamma$ may reach up to a sizeable value $\mathcal{O}\left(10^{-5}\right)$.

\section{B. $P$-wave states}

The masses of $1 P$-wave states $B_{c}(1 P)$ might lie in the range of $(6710,6790) \mathrm{MeV}$, which are consistent with the other predictions with potential models [7-11], and the recent lattice calculations [36]. The $1 P$-wave $B_{c}(1 P)$ states mainly decay via the $E 1$ dominate transitions $1 P \rightarrow 1 S$. We have calculated the partial decay widths for the EM transitions $1 P \rightarrow 1 S$; our results compared with some other predictions are listed in Table V. Most of our results are compatible with the predictions in [7,9-11], except our predicted partial decay widths of $\Gamma\left[B_{c}\left(1 P_{1}\right) \rightarrow B_{c} \gamma\right] \simeq$ $35 \mathrm{keV}$ and $\Gamma\left[B_{c}\left(1 P_{1}^{\prime}\right) \rightarrow B_{c}^{*} \gamma\right] \simeq 40 \mathrm{keV}$ are about a factor of 3-5 larger than the predictions in Refs. [9-11]. The $B_{c}\left(1 P_{1}\right)$ and $B_{c}\left(1 P_{1}^{\prime}\right)$ states might be first found in the $B_{c} \gamma$ final state via their radiative transitions. The branching fractions for $B_{c}\left(1 P_{1}\right)$ and $B_{c}\left(1 P_{1}^{\prime}\right)$ decay into $B_{c} \gamma$ are predicted to be

$$
\begin{aligned}
& \operatorname{Br}\left[B_{c}\left(1 P_{1}\right) \rightarrow B_{c} \gamma\right] \sim 33 \%, \\
& \operatorname{Br}\left[B_{c}\left(1 P_{1}^{\prime}\right) \rightarrow B_{c} \gamma\right] \sim 65 \% .
\end{aligned}
$$

While the $B_{c}\left(1^{3} P_{0}\right)$ and $B_{c}\left(1^{3} P_{2}\right)$ states dominantly decay into the $B_{c}^{*} \gamma$ final state with a decay rate of $\sim 100 \%$, they 
TABLE XII. Strong decay properties for the $3 P$-, $4 P$-wave $B_{c}$ states.

\begin{tabular}{|c|c|c|c|c|c|c|c|}
\hline State & Decay mode & $\Gamma_{t h}(\mathrm{MeV})$ & $B_{r}(\%)$ & State & Decay mode & $\Gamma_{t h}(\mathrm{MeV})$ & $B_{r}(\%)$ \\
\hline \multirow[t]{7}{*}{$3^{3} P_{0}(7420)$} & $B D$ & 9.6 & 3.5 & $3^{3} P_{2}(7464)$ & $B D$ & 22 & 11.1 \\
\hline & $B^{*} D^{*}$ & 255 & 93 & & $B^{*} D$ & 16 & 8.1 \\
\hline & $B_{s}^{0} D_{s}^{+}$ & 9.7 & 3.5 & & $B D^{*}$ & 3.4 & 1.7 \\
\hline & & & & & $B^{*} D^{*}$ & 146 & 74 \\
\hline & & & & & $B_{s}^{0} D_{s}^{+}$ & 2.7 & 1.4 \\
\hline & & & & & $B_{s}^{* 0} D_{s}^{+}$ & 7.8 & 4 \\
\hline & Total & 274 & 100 & & Total & 198 & 100 \\
\hline \multirow[t]{5}{*}{$3 P_{1}^{\prime}(7458)$} & $B^{*} D$ & 13.6 & 7.3 & $3 P_{1}(7441)$ & $B^{*} D$ & 9.3 & 4.3 \\
\hline & $B D^{*}$ & 32 & 17.2 & & $B D^{*}$ & 62 & 28.1 \\
\hline & $B^{*} D^{*}$ & 129 & 69.4 & & $B^{*} D^{*}$ & 145 & 65.8 \\
\hline & $B_{s}^{* 0} D_{s}^{+}$ & 11.1 & 6 & & $B_{s}^{* 0} D_{s}^{+}$ & 4.0 & 1.8 \\
\hline & Total & 185 & 100 & & Total & 220 & 100 \\
\hline \multirow{17}{*}{$4^{3} P_{0}(7693)$} & $B D$ & 13.6 & 25.6 & $4^{3} P_{2}(7732)$ & $B D$ & 21.76 & 11.4 \\
\hline & $B^{*} D^{*}$ & 14 & 26.4 & & $B^{*} D$ & 30.1 & 15.8 \\
\hline & $B_{s}^{0} D_{s}^{+}$ & 7.16 & 13.5 & & $B D^{*}$ & 13.9 & 7.3 \\
\hline & $B_{s}^{* 0} D_{s}^{*+}$ & 4.6 & 8.7 & & $B^{*} D^{*}$ & 7.82 & 4.1 \\
\hline & $B\left(1 P^{\prime}\right) D$ & 7.66 & 14.4 & & $B_{s}^{0} D_{s}^{+}$ & 0.84 & 0.4 \\
\hline & $B(1 P) D$ & 0.44 & 0.83 & & $B_{s}^{* 0} D_{s}^{+}$ & 0.01 & 0.005 \\
\hline & $B D(1 P)$ & 0.07 & 0.13 & & $B_{s}^{0} D_{s}^{*+}$ & 2.34 & 1.2 \\
\hline & $B^{*} D\left(1^{3} P_{0}\right)$ & 5.5 & 10.4 & & $B_{s}^{* 0} D_{s}^{*+}$ & 11.1 & 5.8 \\
\hline & & & & & $B\left(1 P^{\prime}\right) D$ & 27.7 & 14.5 \\
\hline & & & & & $B(1 P) D$ & 6.95 & 3.6 \\
\hline & & & & & $B\left(1^{3} P_{2}\right) D$ & 20.2 & 10.6 \\
\hline & & & & & $B\left(1^{3} P_{0}\right) D^{*}$ & 8.8 & 4.6 \\
\hline & & & & & $B D\left(1 P^{\prime}\right)$ & 13.1 & 6.9 \\
\hline & & & & & $B D(1 P)$ & 6.61 & 3.5 \\
\hline & & & & & $B^{*} D\left(1^{3} P_{0}\right)$ & 10.1 & 5.3 \\
\hline & & & & & $B^{*} D(1 P)$ & 9.22 & 4.8 \\
\hline & Total & 53 & 100 & & Total & 190 & 100 \\
\hline \multirow[t]{19}{*}{$4 P_{1}^{\prime}(7727)$} & $B^{*} D$ & 41.6 & 29.1 & $4 P_{1}(7710)$ & $B^{*} D$ & 24.5 & 19.4 \\
\hline & $B D^{*}$ & 11.9 & 8.4 & & $B D^{*}$ & 3.7 & 2.9 \\
\hline & $B^{*} D^{*}$ & 6.55 & 4.6 & & $B^{*} D^{*}$ & 0.86 & 0.7 \\
\hline & $B_{s}^{* 0} D_{s}^{+}$ & 1.42 & 1.0 & & $B_{s}^{* 0} D_{s}^{+}$ & 4.4 & 3.5 \\
\hline & $B_{s}^{0} D_{s}^{*+}$ & 6.2 & 4.3 & & $B_{s}^{0} D_{s}^{*+}$ & 6.78 & 5.4 \\
\hline & $B_{s}^{* 0} D_{s}^{*+}$ & 9.09 & 6.3 & & $B_{s}^{* 0} D_{s}^{*+}$ & 6.66 & 5.3 \\
\hline & $B\left(1^{3} P_{0}\right) D$ & 10.4 & 7.3 & & $B\left(1^{3} P_{0}\right) D$ & 0.002 & 0.002 \\
\hline & $B\left(1 P^{\prime}\right) D$ & 0.003 & 0.002 & & $B\left(1 P^{\prime}\right) D$ & 0.4 & 0.3 \\
\hline & $B(1 P) D$ & 0.01 & 0.01 & & $B(1 P) D$ & 3.32 & 2.6 \\
\hline & $B\left(1^{3} P_{2}\right) D$ & 36.6 & 25.6 & & $B\left(1^{3} P_{2}\right) D$ & 15 & 11.9 \\
\hline & $B\left(1^{3} P_{0}\right) D^{*}$ & 0.02 & 0.01 & & $B\left(1^{3} P_{0}\right) D^{*}$ & 11.8 & 9.4 \\
\hline & $B D\left(1^{3} P_{0}\right)$ & 13.6 & 9.5 & & $B D\left(1^{3} P_{0}\right)$ & 0.1 & 0.08 \\
\hline & $B D\left(1 P^{\prime}\right)$ & 0.009 & 0.006 & & $B D\left(1 P^{\prime}\right)$ & 15.32 & 12.2 \\
\hline & $B D(1 P)$ & 0.05 & 0.03 & & $B D(1 P)$ & 23.03 & 18.3 \\
\hline & $B^{*} D\left(1^{3} P_{0}\right)$ & 0.1 & 0.07 & & $B^{*} D\left(1^{3} P_{0}\right)$ & 10.02 & 8.0 \\
\hline & $B^{*} D(1 P)$ & 0.31 & 0.22 & & & & \\
\hline & $B_{s} D_{s}\left(1^{3} P_{0}\right)$ & 4.75 & 3.3 & & & & \\
\hline & $B_{s}\left(1^{3} P_{0}\right) D_{s}$ & 0.41 & 0.3 & & & & \\
\hline & Total & 143 & 100 & & Total & 126 & 100 \\
\hline
\end{tabular}

have good potential to be found via the radiative decay chains $B_{c}\left(1^{3} P_{0}\right) \rightarrow B_{c}\left(1^{3} S_{1}\right) \gamma \rightarrow B_{c}\left(1^{1} S_{0}\right) \gamma \gamma$ and $B_{c}\left(1^{3} P_{2}\right) \rightarrow$ $B_{c}\left(1^{3} S_{1}\right) \gamma \rightarrow B_{c}\left(1^{1} S_{0}\right) \gamma \gamma$, respectively.

For the $2 P$-wave states $B_{c}(2 P)$, their masses might lie in the range $(7100,7160) \mathrm{MeV}$, which are consistent with the other model predictions in the literature [7-11,15,16]. The masses for $B_{c}\left(2^{3} P_{0}\right)$ and $B_{c}\left(2 P_{1}\right)$ are slightly lower than the $D B$ mass threshold, while $B_{c}\left(2 P_{1}^{\prime}\right)$ and $B_{c}\left(2^{3} P_{2}\right)$ slightly lie above the $D B$ mass threshold. The $B_{c}\left(2^{3} P_{2}\right)$ state mainly decays into the $D B$ channel, while its radiative 
TABLE XIII. Strong decay properties for the $2 D$-, $3 D$-wave $B_{c}$ states.

\begin{tabular}{|c|c|c|c|c|c|c|c|}
\hline State & Decay mode & $\Gamma_{\mathrm{th}}(\mathrm{MeV})$ & $B_{r}(\%)$ & State & Decay mode & $\Gamma_{t h}(\mathrm{MeV})$ & $B_{r}(\%)$ \\
\hline \multirow[t]{6}{*}{$2^{3} D_{1}(7336)$} & $B D$ & 0.55 & 1.0 & \multirow[t]{6}{*}{$2^{3} D_{3}(7348)$} & $B D$ & 41.6 & 22.1 \\
\hline & $B^{*} D$ & 6.24 & 10.9 & & $B^{*} D$ & 50.8 & 26.9 \\
\hline & $B D^{*}$ & 50.1 & 87 & & $B D^{*}$ & 9.29 & 4.9 \\
\hline & $B^{*} D^{*}$ & 0.48 & 0.8 & & $B^{*} D^{*}$ & 87 & 46.1 \\
\hline & $B_{s}^{0} D_{s}^{+}$ & 0.18 & 0.3 & & $B_{s}^{0} D_{s}^{+}$ & 0.013 & 0.01 \\
\hline & Total & 57 & 100 & & Total & 189 & 100 \\
\hline \multirow[t]{4}{*}{$2 D_{2}^{\prime}(7347)$} & $B^{*} D$ & 57.1 & 34.7 & \multirow[t]{4}{*}{$2 D_{2}(7343)$} & $B^{*} D$ & 38.2 & 27 \\
\hline & $B D^{*}$ & 66.8 & 40.7 & & $B D^{*}$ & 89 & 64 \\
\hline & $B^{*} D^{*}$ & 40.4 & 24.6 & & $B^{*} D^{*}$ & 12.3 & 9 \\
\hline & Total & 164 & 100 & & Total & 139 & 100 \\
\hline \multirow[t]{12}{*}{$3^{3} D_{1}(7611)$} & $B D$ & 25.2 & 28.2 & \multirow[t]{12}{*}{$3^{3} D_{3}(7625)$} & $B D$ & 19.3 & 17 \\
\hline & $B^{*} D$ & 5.65 & 6.3 & & $B^{*} D$ & 29.7 & 26.5 \\
\hline & $B D^{*}$ & 0.48 & 0.5 & & $B D^{*}$ & 20.8 & 18.6 \\
\hline & $B^{*} D^{*}$ & 19.5 & 21.9 & & $B^{*} D^{*}$ & 18.4 & 16.4 \\
\hline & $B_{s}^{0} D_{s}^{+}$ & 2.27 & 2.5 & & $B_{s}^{0} D_{s}^{+}$ & 1.45 & 1.3 \\
\hline & $B_{s}^{* 0} D_{s}^{+}$ & 3.16 & 3.5 & & $B_{s}^{* 0} D_{s}^{+}$ & 0.12 & 0.1 \\
\hline & $B_{s}^{0} D_{s}^{*+}$ & 1.82 & 2.0 & & $B_{s}^{0} D_{s}^{*+}$ & 2.94 & 2.6 \\
\hline & $B_{s}^{* 0} D_{s}^{*+}$ & 16.5 & 18.5 & & $B_{s}^{* 0} D_{s}^{*+}$ & 6.6 & 5.9 \\
\hline & $B(1 P) D$ & 0.76 & 0.9 & & $B\left(1 P^{\prime}\right) D$ & 0.001 & 0.001 \\
\hline & $B^{*} D\left(1^{3} P_{0}\right)$ & 13.9 & 15.6 & & $B(1 P) D$ & 4.62 & 4.1 \\
\hline & & & & & $B^{*} D\left(1^{3} P_{0}\right)$ & 8.14 & 7.3 \\
\hline & Total & 89 & 100 & & Total & 112 & 100 \\
\hline \multirow[t]{11}{*}{$3 D_{2}^{\prime}(7623)$} & $B^{*} D$ & 45.8 & 34.6 & \multirow[t]{11}{*}{$3 D_{2}(7620)$} & $B^{*} D$ & 38.9 & 34.2 \\
\hline & $B D^{*}$ & 20.6 & 15.6 & & $B D^{*}$ & 13.8 & 12 \\
\hline & $B^{*} D^{*}$ & 21.1 & 16 & & $B^{*} D^{*}$ & 22.1 & 19 \\
\hline & $B_{s}^{* 0} D_{s}^{+}$ & 2.25 & 1.7 & & $B_{s}^{* 0} D_{s}^{+}$ & 3.89 & 3.4 \\
\hline & $B_{s}^{0} D_{s}^{*+}$ & 6.33 & 4.8 & & $B_{s}^{0} D_{s}^{*+}$ & 6.46 & 5.7 \\
\hline & $B_{s}^{* 0} D_{s}^{*+}$ & 9.07 & 6.8 & & $B_{s}^{* 0} D_{s}^{*+}$ & 11.6 & 10 \\
\hline & $B\left(1^{3} P_{0}\right) D$ & 12.1 & 9.1 & & $B\left(1^{3} P_{0}\right) D$ & 0.03 & 0.03 \\
\hline & $B(1 P) D$ & 0.02 & 0.02 & & $B(1 P) D$ & 2.82 & 2.5 \\
\hline & $B D\left(1^{3} P_{0}\right)$ & 14.4 & 10.9 & & $B D\left(1^{3} P_{0}\right)$ & 0.65 & 0.6 \\
\hline & $B^{*} D\left(1^{3} P_{0}\right)$ & 0.65 & 0.5 & & $B^{*} D\left(1^{3} P_{0}\right)$ & 13.6 & 12 \\
\hline & Total & 132 & 100 & & Total & 114 & 100 \\
\hline
\end{tabular}

decay rates into the $B_{c}\left(n^{3} S_{1}\right) \gamma(n=1,2)$ are also sizeable. Their partial widths are predicted to be

$$
\begin{gathered}
\Gamma\left[B_{c}\left(2^{3} P_{2}\right) \rightarrow D B\right] \simeq 760 \mathrm{keV}, \\
\Gamma\left[B_{c}\left(2^{3} P_{2}\right) \rightarrow B_{c}^{*} \gamma\right] \simeq 52 \mathrm{keV}, \\
\Gamma\left[B_{c}\left(2^{3} P_{2}\right) \rightarrow B_{c}^{*}(2 S) \gamma\right] \simeq 50 \mathrm{keV} .
\end{gathered}
$$

Thus, the total width of $B_{c}\left(2^{3} P_{2}\right)$ is $\Gamma_{\text {total }}\left[B_{c}\left(2^{3} P_{2}\right)\right] \simeq$ $880 \mathrm{keV}$. The $B_{c}\left(2^{3} P_{2}\right)$ state may have potential to be observed in the $D B$ and $B_{c} \gamma$ final states, while for the $B_{c}\left(2^{3} P_{0}\right), B_{c}\left(2 P_{1}\right)$, and $B_{c}\left(2 P_{1}^{\prime}\right)$ states, their decays are governed by the EM transitions. The radiative decay properties of these states have been given in Table VII. With these predictions, the total widths for $B_{c}\left(2^{3} P_{0}\right), B_{c}\left(2 P_{1}\right)$, and $B_{c}\left(2 P_{1}^{\prime}\right)$ are estimated to be $\Gamma_{\text {total }}\left[B_{c}\left(2^{3} P_{0}\right)\right] \simeq 100 \mathrm{keV}$,
$\Gamma_{\text {total }}\left[B_{c}\left(2 P_{1}\right)\right] \simeq 120 \mathrm{keV}$, and $\Gamma_{\text {total }}\left[B_{c}\left(2 P_{1}^{\prime}\right)\right] \simeq 133 \mathrm{keV}$, respectively. The branching fractions for $B_{c}\left(2 P_{1}\right) \rightarrow B_{c} \gamma$, $B_{c}\left(2 P_{1}^{\prime}\right) \rightarrow B_{c} \gamma$ and $B_{c}\left(2^{3} P_{0}\right) \rightarrow B_{c}^{*} \gamma$ are predicted to be

$$
\begin{aligned}
& \operatorname{Br}\left[B_{c}\left(2 P_{1}\right) \rightarrow B_{c} \gamma\right] \simeq 20 \%, \\
& \operatorname{Br}\left[B_{c}\left(2 P_{1}^{\prime}\right) \rightarrow B_{c} \gamma\right] \simeq 33 \% \\
& \operatorname{Br}\left[B_{c}\left(2^{3} P_{0}\right) \rightarrow B_{c}^{*} \gamma\right] \simeq 41 \% .
\end{aligned}
$$

The large branching fractions indicate that $B_{c}\left(2 P_{1}\right)$ and $B_{c}\left(2 P_{1}^{\prime}\right)$ may be established in the $B_{c} \gamma$ channel, while $B_{c}\left(2^{3} P_{0}\right)$ may be observed via the radiative decay chain $B_{c}\left(2^{3} P_{0}\right) \rightarrow B_{c}^{*} \gamma \rightarrow B_{c} \gamma \gamma$. It should be pointed out that the $B_{c}\left(2 P_{1}\right), B_{c}\left(2 P_{1}^{\prime}\right)$, and $B_{c}\left(2^{3} P_{2}\right)$ states may lie above the $B^{*} D$ threshold, so they may have fairly large strong decay 
TABLE XIV. Strong decay properties for the $1 F$-, $2 F$-wave $B_{c}$ states.

\begin{tabular}{|c|c|c|c|c|c|c|c|}
\hline State & Decay mode & $\Gamma_{t h}(\mathrm{MeV})$ & $B_{r}(\%)$ & State & Decay mode & $\Gamma_{t h}(\mathrm{MeV})$ & $B_{r}(\%)$ \\
\hline \multirow[t]{3}{*}{$1^{3} F_{2}(7235)$} & $B D$ & 61.9 & 85 & \multirow[t]{3}{*}{$1^{3} F_{4}(7227)$} & $B D$ & 0.85 & 97 \\
\hline & $B^{*} D$ & 11.1 & 15 & & $B^{*} D$ & 0.03 & 3 \\
\hline & Total & 73 & 100 & & Total & 0.88 & 100 \\
\hline \multirow{2}{*}{$1 F_{3}^{\prime}(7240)$} & $B^{*} D$ & 15.1 & 100 & \multirow{2}{*}{$1 F_{3}(7224)$} & $B^{*} D$ & 8.53 & 100 \\
\hline & Total & 15.1 & 100 & & Total & 8.53 & 100 \\
\hline \multirow[t]{8}{*}{$2^{3} F_{2}(7518)$} & $B D$ & 45.1 & 20.2 & \multirow[t]{8}{*}{$2^{3} F_{4}(7514)$} & $B D$ & 8 & 6 \\
\hline & $B^{*} D$ & 19.2 & 8.6 & & $B^{*} D$ & 20.9 & 16 \\
\hline & $B D^{*}$ & 0.39 & 0.2 & & $B D^{*}$ & 37.7 & 29 \\
\hline & $B^{*} D^{*}$ & 151 & 68 & & $B^{*} D^{*}$ & 57 & 43 \\
\hline & $B_{s}^{0} D_{s}^{+}$ & 0.68 & 0.3 & & $B_{s}^{0} D_{s}^{+}$ & 4.48 & 3.4 \\
\hline & $B_{s}^{* 0} D_{s}^{+}$ & 3.63 & 1.6 & & $B_{s}^{* 0} D_{s}^{+}$ & 3.26 & 2.5 \\
\hline & $B_{s}^{0} D_{s}^{*+}$ & 3.17 & 1.4 & & $B_{s}^{0} D_{s}^{*+}$ & 0.05 & 0.04 \\
\hline & Total & 223 & 100 & & Total & 131 & 100 \\
\hline \multirow[t]{6}{*}{$2 F_{3}^{\prime}(7525)$} & $B^{*} D$ & 45.2 & 25 & \multirow[t]{6}{*}{$2 F_{3}(7508)$} & $B^{*} D$ & 43.9 & 25 \\
\hline & $B D^{*}$ & 41.0 & 23 & & $B D^{*}$ & 30.2 & 17 \\
\hline & $B^{*} D^{*}$ & 80.3 & 45 & & $B^{*} D^{*}$ & 90.2 & 52 \\
\hline & $B_{s}^{* 0} D_{s}^{+}$ & 7.19 & 4 & & $B_{s}^{* 0} D_{s}^{+}$ & 7.78 & 4.5 \\
\hline & $B_{s}^{0} D_{s}^{*+}$ & 4.53 & 3 & & $B_{s}^{0} D_{s}^{*+}$ & 2.57 & 1.5 \\
\hline & Total & 178 & 100 & & Total & 175 & 100 \\
\hline
\end{tabular}

widths $\mathcal{O}(10-100) \mathrm{MeV}$ into $B^{*} D$ and/or $B D$ channels as predicted in Ref. [17].

For the higher $P$-wave states $B_{c}(n P)(n=3,4)$, many OZI-allowed strong decay channels are open (see Table XII); thus, these states usually are broad states with a width of $\mathcal{O}(100) \mathrm{MeV}$, except the $B_{c}\left(4^{3} P_{0}\right)$ state which has a relatively narrow width of $\mathcal{O}(10) \mathrm{MeV}$. The $B_{c}\left(4^{3} P_{0}\right)$ state may be first observed in the $D B$ channel; the branching fraction for the process $B_{c}\left(4^{3} P_{0}\right) \rightarrow D B$ can reach up to $\sim 20 \%$.

\section{C. $D$-wave states}

The masses of the $1 D$-wave states $B_{c}(1 D)$ are predicted to be $\sim 7.02 \mathrm{GeV}$ in this work. The mass splitting between the $1 D$-wave states is no more than $15 \mathrm{MeV}$. The masses predicted by us are consistent with the results in Refs. [7,8,11]. The $1 D$-wave states mainly decay via the EM transitions, which have been given in Table V. It is seen that our main results are in reasonable agreement with the other predictions. Our study indicates that the $B_{c}\left(1^{3} D_{3}\right)$ state may have a relatively large potential to be observed via the radiative decay chain $B_{c}\left(1^{3} D_{3}\right) \rightarrow B_{c}\left(1^{3} P_{2}\right) \gamma \rightarrow B_{c}\left(1^{3} S_{1}\right) \gamma \gamma \rightarrow$ $B_{c}\left(1^{1} S_{0}\right) \gamma \gamma \gamma$, and the branching fraction for this chain is estimated to be $\sim 100 \%$. The optimal decay chain for the observations of $B_{c}\left(1^{3} D_{1}\right)$ is $B_{c}\left(1^{3} D_{1}\right) \rightarrow B_{c}\left(1^{3} P_{0}\right) \gamma \rightarrow$ $B_{c}\left(1^{3} S_{1}\right) \gamma \gamma \rightarrow B_{c}\left(1^{1} S_{0}\right) \gamma \gamma \gamma$, and the branching fraction for this chain is estimated to be $\sim 60 \%$. The optimal decay chains for the observations of $B_{c}\left(1 D_{2}\right)$ are $B_{c}\left(1 D_{2}\right) \rightarrow$ $B_{c}\left(1 P_{1}\right) \gamma \rightarrow B_{c}\left(1^{3} S_{1}\right) \gamma \gamma \rightarrow B_{c}\left(1^{1} S_{0}\right) \gamma \gamma \gamma$ and $B_{c}\left(1 D_{2}\right) \rightarrow$ $B_{c}\left(1 P_{1}\right) \gamma \rightarrow B_{c}\left(1^{1} S_{0}\right) \gamma \gamma$, and the branching fractions for these chains are estimated to be $\sim 50 \%$ and $\sim 30 \%$, respectively. For the observations of $B_{c}\left(1 D_{2}^{\prime}\right)$, the optimal decay chains are $B_{c}\left(1 D_{2}^{\prime}\right) \rightarrow B_{c}\left(1 P_{1}^{\prime}\right) \gamma \rightarrow B_{c}\left(1^{3} S_{1}\right) \gamma \gamma \rightarrow$ $B_{c}\left(1^{1} S_{0}\right) \gamma \gamma \gamma$ and $B_{c}\left(1 D_{2}^{\prime}\right) \rightarrow B_{c}\left(1 P_{1}^{\prime}\right) \gamma \rightarrow B_{c}\left(1^{1} S_{0}\right) \gamma \gamma$, and the branching fractions for these chains are estimated to be $\sim 35 \%$ and $\sim 47 \%$, respectively.

The masses of the $2 D$ states are predicted to be $\sim 7.34 \mathrm{GeV}$, which is very close to the $D_{s} B_{s}$ threshold. Their decays are governed by the strong decay modes, such as $D B, D B^{*}, B D^{*}$, or $B^{*} D^{*}$. Their strong decay properties predicted by us have been listed in Table XIII. There are few discussions about the radiative decays of the $2 D$-wave $B_{c}$ states in the literature. In this work, we also calculate their radiative decay properties; our results are given in Table VI. It is found that the $B_{c}\left(2^{3} D_{1}\right)$ state has a relatively narrow width of $\Gamma \sim 58 \mathrm{MeV}$. The decays of $B_{c}\left(2^{3} D_{1}\right)$ are governed by the $B D^{*}$ mode with a branching fraction

$$
\operatorname{Br}\left[B_{c}\left(2^{3} D_{1}\right) \rightarrow B D^{*}\right] \simeq 87 \% .
$$

The other three $2 D$ states $B_{c}\left(2^{3} D_{3}\right), B_{c}\left(2 D_{2}\right)$, and $B_{c}\left(2 D_{2}^{\prime}\right)$ are broad states with a width of $\sim 100-200 \mathrm{MeV}$. The $B_{c}\left(2^{3} D_{3}\right)$ state mainly decays into $D B, D B^{*}$, and $B^{*} D^{*}$ channels. While the $B_{c}\left(2 D_{2}\right)$ and $B_{c}\left(2 D_{2}^{\prime}\right)$ states dominantly decay into $D B^{*}, B D^{*}$, or $B^{*} D^{*}$ channels. Combing the strong and radiative decay properties with each other, it is found that the branching fractions of the dominant EM decay processes $B_{c}(2 D) \rightarrow B_{c}(n P)(n=1$, 2) are $\mathcal{O}\left(10^{-4}\right)$. The observations of the $D B, D B^{*}, B D^{*}$, or $B^{*} D^{*}$ final states might be useful to search for these missing $2 D$ states in future experiments. 
TABLE XV. Strong decay properties for the $3 F$-wave $B_{c}$ states.

\begin{tabular}{|c|c|c|c|c|c|c|c|}
\hline State & Decay mode & $\Gamma_{t h}(\mathrm{MeV})$ & $B_{r}(\%)$ & State & Decay mode & $\Gamma_{t h}(\mathrm{MeV})$ & $B_{r}(\%)$ \\
\hline \multirow[t]{23}{*}{$3^{3} F_{2}(7730)$} & $B D$ & 32.1 & 14 & $3^{3} F_{4}(7771)$ & $B D$ & 2.82 & 1.6 \\
\hline & $B^{*} D$ & 16.1 & 7 & & $B^{*} D$ & 8.9 & 5.0 \\
\hline & $B D^{*}$ & 3.89 & 1.7 & & $B D^{*}$ & 20.2 & 11.4 \\
\hline & $B^{*} D^{*}$ & 72 & 31.5 & & $B^{*} D^{*}$ & 50.9 & 28.7 \\
\hline & $B_{s}^{0} D_{s}^{+}$ & 0.38 & 0.2 & & $B_{s}^{0} D_{s}^{+}$ & 3.2 & 1.8 \\
\hline & $B_{s}^{* 0} D_{s}^{+}$ & 0.11 & 0.05 & & $B_{s}^{* 0} D_{s}^{+}$ & 3.2 & 1.8 \\
\hline & $B_{s}^{0} D_{s}^{*+}$ & 2.09 & 0.9 & & $B_{s}^{0} D_{s}^{*+}$ & 0.4 & 0.2 \\
\hline & $B_{s}^{* 0} D_{s}^{*+}$ & 5.25 & 2.3 & & $B_{s}^{* 0} D_{s}^{*+}$ & 9.19 & 5.2 \\
\hline & $B\left(1 P^{\prime}\right) D$ & 19.5 & 8.5 & & $B\left(1 P^{\prime}\right) D$ & 19.4 & 10.9 \\
\hline & $B(1 P) D$ & 5.03 & 2.2 & & $B(1 P) D$ & 1.85 & 1.04 \\
\hline & $B\left(1^{3} P_{2}\right) D$ & 12.1 & 5.3 & & $B\left(1^{3} P_{2}\right) D$ & 12.3 & 6.9 \\
\hline & $B\left(1^{3} P_{0}\right) D^{*}$ & 2.56 & 1.1 & & $B\left(1^{3} P_{0}\right) D^{*}$ & 6.82 & 3.8 \\
\hline & $B D\left(1 P^{\prime}\right)$ & 45.8 & 20 & & $B\left(1 P^{\prime}\right) D^{*}$ & 0.02 & 0.01 \\
\hline & $B D(1 P)$ & 2.3 & 1 & & $B(1 P) D^{*}$ & 3.03 & 1.7 \\
\hline & $B^{*} D\left(1^{3} P_{0}\right)$ & 9.14 & 4 & & $B D\left(1 P^{\prime}\right)$ & 9.19 & 5.2 \\
\hline & $B^{*} D(1 P)$ & 0.48 & 0.2 & & $B D(1 P)$ & 11.2 & 6.3 \\
\hline & & & & & $B D\left(1^{3} P_{2}\right)$ & 1.61 & 0.9 \\
\hline & & & & & $B^{*} D\left(1^{3} P_{0}\right)$ & 1.91 & 1.1 \\
\hline & & & & & $B^{*} D\left(1 P^{\prime}\right)$ & 2.83 & 1.6 \\
\hline & & & & & $B^{*} D(1 P)$ & 8.42 & 4.7 \\
\hline & & & & & $B_{s}(1 P) D_{s}$ & $<0.0001$ & $\simeq 0$ \\
\hline & & & & & $B_{s}^{*} D_{s}\left(1^{3} P_{0}\right)$ & 0.02 & 0.01 \\
\hline & Total & 228 & 100 & & Total & 177 & 100 \\
\hline \multirow[t]{26}{*}{$3 F_{3}^{\prime}(7779)$} & $B^{*} D$ & 33.6 & 11 & $3 F_{3}(7768)$ & $B^{*} D$ & 39.9 & 12 \\
\hline & $B D^{*}$ & 34.4 & 11.3 & & $B D^{*}$ & 31.3 & 9.5 \\
\hline & $B^{*} D^{*}$ & 59.9 & 19.6 & & $B^{*} D^{*}$ & 63.5 & 19.3 \\
\hline & $B_{s}^{* 0} D_{s}^{+}$ & 4.2 & 1.4 & & $B_{s}^{* 0} D_{s}^{+}$ & 2.64 & 0.8 \\
\hline & $B_{s}^{0} D_{s}^{*+}$ & 1.69 & 0.6 & & $B_{s}^{0} D_{s}^{*+}$ & 2.02 & 0.6 \\
\hline & $B_{s}^{* 0} D_{s}^{*+}$ & 4.85 & 1.6 & & $B_{s}^{* 0} D_{s}^{*+}$ & 3.63 & 1.1 \\
\hline & $B\left(1^{3} P_{0}\right) D$ & 0.008 & 0.003 & & $B\left(1^{3} P_{0}\right) D$ & 0.01 & 0.003 \\
\hline & $B\left(1 P^{\prime}\right) D$ & 0.01 & 0.003 & & $B\left(1 P^{\prime}\right) D$ & 6.25 & 1.9 \\
\hline & $B(1 P) D$ & $<0.001$ & $\simeq 0$ & & $B(1 P) D$ & 2.26 & 0.7 \\
\hline & $B\left(1^{3} P_{2}\right) D$ & 36.7 & 12 & & $B\left(1^{3} P_{2}\right) D$ & 27.6 & 8.4 \\
\hline & $B\left(1^{3} P_{0}\right) D^{*}$ & 0.08 & 0.03 & & $B\left(1^{3} P_{0}\right) D^{*}$ & 8.06 & 2.5 \\
\hline & $B\left(1 P^{\prime}\right) D^{*}$ & 30.4 & 10 & & $B\left(1 P^{\prime}\right) D^{*}$ & 8.69 & 2.6 \\
\hline & $B(1 P) D^{*}$ & 7.75 & 2.5 & & $B(1 P) D^{*}$ & 2.3 & 0.7 \\
\hline & $B\left(1^{3} P_{2}\right) D^{*}$ & 0.68 & 0.2 & & $B D\left(1^{3} P_{0}\right)$ & 0.6 & 0.2 \\
\hline & $B D\left(1^{3} P_{0}\right)$ & 0.11 & 0.04 & & $B D\left(1 P^{\prime}\right)$ & 11.6 & 3.5 \\
\hline & $B D\left(1 P^{\prime}\right)$ & 0.07 & 0.02 & & $B D(1 P)$ & 16.6 & 5.1 \\
\hline & $B D(1 P)$ & 0.56 & 0.2 & & $B D\left(1^{3} P_{2}\right)$ & 34.2 & 10 \\
\hline & $B D\left(1^{3} P_{2}\right)$ & 27.1 & 8.9 & & $B^{*} D\left(1^{3} P_{0}\right)$ & 1.73 & 0.53 \\
\hline & $B^{*} D\left(1^{3} P_{0}\right)$ & 0.13 & 0.04 & & $B^{*} D\left(1 P^{\prime}\right)$ & 57.4 & 17.5 \\
\hline & $B^{*} D\left(1 P^{\prime}\right)$ & 38.9 & 12.8 & & $B^{*} D(1 P)$ & 8.23 & 2.5 \\
\hline & $B^{*} D(1 P)$ & 19.2 & 6.3 & & $B_{s}\left(1^{3} P_{0}\right) D_{s}$ & 0.003 & $\simeq 0$ \\
\hline & $B_{s}\left(1^{3} P_{0}\right) D_{s}$ & 1.38 & 0.45 & & $B_{s} D_{s}\left(1^{3} P_{0}\right)$ & 0.14 & 0.04 \\
\hline & $B_{s}(1 P) D_{s}$ & $<0.0001$ & $\simeq 0$ & & $B_{s}^{*} D_{s}\left(1^{3} P_{0}\right)$ & 0.01 & 0.003 \\
\hline & $B_{s} D_{s}\left(1^{3} P_{0}\right)$ & 3.03 & 1.0 & & & & \\
\hline & $B_{s}^{*} D_{s}\left(1^{3} P_{0}\right)$ & 0.01 & 0.003 & & & & \\
\hline & Total & 305 & 100 & & Total & 329 & 100 \\
\hline
\end{tabular}

The higher $3 D$-wave states $B_{c}(3 D)$ are also studied in the present work. The masses predicted by us are about $7.62 \mathrm{GeV}$, which are comparable with those predicted in Ref. [8], while they are about $150 \mathrm{MeV}$ smaller than those predicted in Refs. [15,16]. The strong decay properties are shown in Table XIII. It is found that these higher $3 D$-wave states have a width of $\sim 100 \mathrm{MeV}$. These higher states might be observed in their dominant strong decay channels. 


\section{D. $F$-wave states}

The masses of the $1 F$-wave states $B_{c}\left(1^{3} F_{4}\right), B_{c}\left(1 F_{3}\right)$, $B_{c}\left(1 F_{3}^{\prime}\right)$, and $B_{c}\left(1^{3} F_{2}\right)$ are predicted to be $\sim 7.23 \mathrm{GeV}$, which are comparable to those predicted in Refs. $[8,11,16]$. These $1 F$ wave states lie above the mass threshold of $D B$ and $B^{*} D$, while below the $D^{*} B$ threshold. From our predictions of the strong decay properties for these $1 F$ wave states (see Table XIV), it is found that the $B_{c}\left(1^{3} F_{4}\right)$ state might be a very narrow state with a width of $\sim 1 \mathrm{MeV}$, its decays are governed by the $D B$ mode. Both $B_{c}\left(1 F_{3}\right)$ and $B_{c}\left(1 F_{3}^{\prime}\right)$ are narrow states with a width of $\sim 10 \mathrm{MeV}$, they dominantly decay into the $D B^{*}$ channel. The $B_{c}\left(1^{3} F_{2}\right)$ should be a relatively broad state with a width of $\sim 70 \mathrm{MeV}$; it mainly decays into the $D B$ channel with a branching fraction of $\operatorname{Br}\left[B_{c}\left(1^{3} F_{2}\right) \rightarrow D B\right] \simeq 85 \%$. To look for the missing $1 F$-wave $B_{c}$ states, the $D B$ and $B^{*} D$ final states are worth observing.

The predicted masses for the $2 F$ - and $3 F$-wave $B_{c}$ states are $\sim 7.5 \mathrm{GeV}$ and $\sim 7.8 \mathrm{GeV}$, respectively, which are comparable with the predictions in Refs. $[8,11]$. There are many strong decay channels for these higher mass $F$ wave states. Our predictions of their strong decay properties have been listed in Tables XIV and XV. It is found that the higher mass $F$-wave states might be broad states with a width of $\sim 100-300 \mathrm{MeV}$.

\section{SUMMARY}

In this paper, we have calculated the $B_{c}$ meson spectrum up to the $6 S$ states with a nonrelativistic linear potential model by further constraining the model parameters with the mass of $B_{c}(2 S)$ newly measured by the CMS Collaboration. As important tasks of this work, the radiative transitions between the $B_{c}$ states and the OZI-allowed two-body strong decays for the higher mass excited $B_{c}$ states are evaluated with the wave functions obtained from the linear potential model. Our calculations may provide useful references to search for the excited $B_{c}$ states. The main results are emphasized as follows.

For the $S$-wave states, the $2 S$ hyperfine splitting is predicted to be $m\left[B_{c}^{*}(2 S)\right]-m\left[B_{c}(2 S)\right] \simeq 19 \mathrm{MeV}$. The mass of the newly observed $B_{c}^{*}(2 S)$ state might be determined via the $M 1$ transition $B_{c}^{*}(2 S) \rightarrow B_{c} \gamma$ in future experiments. The $3 S$-wave states $B_{c}\left(3^{1} S_{0}\right)$ and $B_{c}\left(3^{3} S_{1}\right)$ are about $50 \mathrm{MeV}$ above the $D B^{*}$ threshold; their widths are estimated to be $\sim 100 \mathrm{MeV}$. Since production rates of the $3 S$-wave $B_{c}$ states in $p p$ collisions at the LHC are comparable with those of the $2 S$-wave $B_{c}$ states [18], both $B_{c}\left(3^{1} S_{0}\right)$ and $B_{c}\left(3^{3} S_{1}\right)$ states may have large possibilities to be established in the $D B^{*}$ final state, while $B_{c}\left(3^{3} S_{1}\right)$ might be observed in the $D B$ final state as well.

For the $P$-wave states, it is found that the decays of the $2 P$-wave states, $B_{c}\left(2^{3} P_{0}\right), B_{c}\left(2 P_{1}\right)$, and $B_{c}\left(2 P_{1}^{\prime}\right)$ together with all of the $1 P$-wave states are governed by the $E 1$ transitions; their typical decay widths are $\sim 100 \mathrm{keV}$. It should be possible to observe these $P$-wave states via their dominant radiative decay processes with the higher statistics of the LHC. The $B_{c}\left(2^{3} P_{2}\right)$ state is just $\sim 20 \mathrm{MeV}$ above the $D B$ threshold. It mainly decays into the $D B$ channel with a very narrow width of $\Gamma \sim 1 \mathrm{MeV}$, so it has a large potential to be first observed in the $D B$ final state. The predicted masses of $3 P$-wave states are in the range of (7420, 7470) MeV. They are broad states with widths of $\sim 200 \mathrm{MeV}$, and strongly couple to the $B^{*} D^{*}$ final state. It is interesting to find that the $4 P$-wave states $B_{c}\left(4^{3} P_{0}\right)$, $B_{c}\left(4 P_{1}\right)$, and $B_{c}\left(4 P_{1}^{\prime}\right)$ with a mass around $7.7 \mathrm{GeV}$ may have relatively narrow widths of $\mathcal{O}(100) \mathrm{MeV}$; these higher $P$-wave states might be first observed in their dominant channel $D B$ or $D B^{*}$.

The $1 D$-wave states mainly decay via the EM transitions. Our study indicates that these $1 D$-wave states may have a relatively large potential to be observed via the radiative decay chains. For example, to look for the $B_{c}\left(1^{3} D_{3}\right)$ state, the $B_{c}\left(1^{3} D_{3}\right) \rightarrow B_{c}\left(1^{3} P_{2}\right) \gamma \rightarrow B_{c}\left(1^{3} S_{1}\right) \gamma \gamma \rightarrow B_{c}\left(1^{1} S_{0}\right) \gamma \gamma \gamma$ is worthy to be searched, for the branching fraction of this chain is estimated to be $\sim 100 \%$. The masses of the $2 D$ and $3 D$ states are predicted to be $\sim 7.34$ and $7.62 \mathrm{GeV}$, respectively. Their decays are governed by the strong decay modes, such as $D B, D B^{*}, B D^{*}$, or $B^{*} D^{*}$. These higher $D$ wave states usually have a width of $\mathcal{O}(100) \mathrm{MeV}$. The observations of the $D B, D B^{*}, B D^{*}$, or $B^{*} D^{*}$ final states might be useful to search for these missing $2 D$ and $3 D$ states in future experiments.

For the $F$-wave states, one should pay more attention to $1 F$-wave $B_{c}$ states in future observations. They have a mass of $\sim 7.23 \mathrm{GeV}$ and lie between the $D B$ and $B^{*} D$ mass thresholds. They are narrow states with a width of several $\mathrm{MeV}$ to several ten $\mathrm{MeV}$, and dominantly decay into $D B$ or $B^{*} D$ channels. For example, the $B_{c}\left(1^{3} F_{4}\right)$ state might be a very narrow state with a width of $\sim 1 \mathrm{MeV}$; its decays are governed by the $D B$ mode. To look for the missing $1 F$-wave $B_{c}$ states, the $D B$ and $B^{*} D$ final states are worth observing.

Finally, it should be pointed out the strong decay widths of the excited $B_{c}$ states predicted in this work may have large uncertainties, for the parameter $\gamma$ cannot be directly determined by the strong decay processes of $B_{c}$ states. Fortunately, the uncertainties of the total strong decay widths of the excited $B_{c}$ states do not affect the important information, such as the dominant decay modes and corresponding decay rates, for our searching for the excited $B_{c}$ states in future experiments. Furthermore, the mixing angles for ${ }^{3} P_{1}-{ }^{1} P_{1},{ }^{3} D_{2}-{ }^{1} D_{2}$, and ${ }^{3} F_{3}-{ }^{1} F_{3}$ have obvious model dependencies. The uncertainties of the mixing angles also affect our predictions of the decay properties of the mixed states.

\section{ACKNOWLEDGMENTS}

This work is supported by the National Natural Science Foundation of China under Grants No. 11775078, No. U1832173, No. 11705056, and No. 11405053. 
[1] E. Eichten and F. Feinberg, Spin dependent forces in QCD, Phys. Rev. D 23, 2724 (1981).

[2] F. Abe et al. (CDF Collaboration), Observation of $B_{c}$ mesons in $p \bar{p}$ collisions at $\sqrt{s}=1.8 \mathrm{TeV}$, Phys. Rev. D 58, 112004 (1998).

[3] G. Aad et al. (ATLAS Collaboration), Observation of an Excited $B_{c}^{ \pm}$Meson State with the ATLAS Detector, Phys. Rev. Lett. 113, 212004 (2014).

[4] R. Aaij et al. (LHCb Collaboration), Search for excited $B_{c}^{+}$ states, J. High Energy Phys. 01 (2018) 138.

[5] A. M. Sirunyan et al. (CMS Collaboration), Observation of Two Excited $B_{c}^{+}$States and Measurement of the $B_{c}^{+}(2 S)$ Mass in pp Collisions at $\sqrt{s}=13 \mathrm{TeV}$, Phys. Rev. Lett. 122, 132001 (2019).

[6] S. Godfrey and N. Isgur, Mesons in a relativized quark model with chromodynamics, Phys. Rev. D 32, 189 (1985).

[7] E. J. Eichten and C. Quigg, Mesons with beauty and charm: Spectroscopy, Phys. Rev. D 49, 5845 (1994).

[8] J. Zeng, J. W. Van Orden, and W. Roberts, Heavy mesons in a relativistic model, Phys. Rev. D 52, 5229 (1995).

[9] V. V. Kiselev, A. K. Likhoded, and A. V. Tkabladze, $B_{c}$ spectroscopy, Phys. Rev. D 51, 3613 (1995).

[10] D. Ebert, R. N. Faustov, and V. O. Galkin, Properties of heavy quarkonia and $B_{c}$ mesons in the relativistic quark model, Phys. Rev. D 67, 014027 (2003).

[11] S. Godfrey, Spectroscopy of $B_{c}$ mesons in the relativized quark model, Phys. Rev. D 70, 054017 (2004).

[12] L. P. Fulcher, Phenomenological predictions of the properties of the $B_{c}$ system, Phys. Rev. D 60, 074006 (1999).

[13] A. Abd El-Hady, J. R. Spence, and J. P. Vary, Radiative decays of $B_{c}$ mesons in a Bethe-Salpeter model, Phys. Rev. D 71, 034006 (2005).

[14] N. Devlani, V. Kher, and A. K. Rai, Masses and electromagnetic transitions of the $B_{c}$ mesons, Eur. Phys. J. A 50, 154 (2014).

[15] A. P. Monteiro, M. Bhat, and K. B. Vijaya Kumar, Mass spectra and decays of ground and orbitally excited $c \bar{b}$ states in nonrelativistic quark model, Int. J. Mod. Phys. A 32, 1750021 (2017).

[16] N. R. Soni, B. R. Joshi, R. P. Shah, H. R. Chauhan, and J. N. Pandya, $Q \bar{Q}(Q \in\{b, c\})$ spectroscopy using the Cornell potential, Eur. Phys. J. C 78, 592 (2018).

[17] A. P. Monteiro, M. Bhat, and K. B. Vijaya Kumar, $c \bar{b}$ spectrum and decay properties with coupled channel effects, Phys. Rev. D 95, 054016 (2017).

[18] E. J. Eichten and C. Quigg, Mesons with beauty and charm: New horizons in spectroscopy, Phys. Rev. D 99, 054025 (2019).

[19] M. Baldicchi and G. M. Prosperi, $B_{c}$ meson and the lightheavy quarkonium spectrum, Phys. Rev. D 62, 114024 (2000).

[20] S. Tang, Y. Li, P. Maris, and J. P. Vary, $B_{c}$ mesons and their properties on the light front, Phys. Rev. D 98, 114038 (2018).

[21] S. M. Ikhdair and R. Sever, $B_{c}$ meson spectrum and hyperfine splittings in the shifted large $N$ expansion technique, Int. J. Mod. Phys. A 18, 4215 (2003).

[22] S. M. Ikhdair and R. Sever, Spectroscopy of $B_{c}$ meson in a semirelativistic quark model using the shifted large $N$ expansion method, Int. J. Mod. Phys. A 19, 1771 (2004).
[23] D. M. Li, B. Ma, Y. X. Li, Q. K. Yao, and H. Yu, Meson spectrum in Regge phenomenology, Eur. Phys. J. C 37, 323 (2004).

[24] K. W. Wei and X. H. Guo, Mass spectra of doubly heavy mesons in Regge phenomenology, Phys. Rev. D 81, 076005 (2010).

[25] X. H. Guo, K. W. Wei, and X. H. Wu, Some mass relations for mesons and baryons in Regge phenomenology, Phys. Rev. D 78, 056005 (2008).

[26] A. M. Badalian, B. L. G. Bakker, and I. V. Danilkin, The hyperfine splittings in bottomonium and the $B_{q}(q=n, s, c)$ mesons, Phys. Rev. D 81, 071502 (2010); Erratum 81, 099902(E) (2010).

[27] Z. G. Wang, Analysis of the vector and axialvector $B_{c}$ mesons with QCD sum rules, Eur. Phys. J. A 49, 131 (2013).

[28] W. Chen, Z. X. Cai, and S. L. Zhu, Masses of the tensor mesons with $J^{P}=2^{-}$, Nucl. Phys. B887, 201 (2014).

[29] A. K. Rai, B. Patel, and P. C. Vinodkumar, Properties of $Q \bar{Q}$ mesons in non-relativistic QCD formalism, Phys. Rev. C 78, 055202 (2008).

[30] B. Patel and P. C. Vinodkumar, Properties of $Q \bar{Q}(Q \in b, c)$ mesons in Coulomb plus Power potential, J. Phys. G 36, 035003 (2009).

[31] A. Bernotas and V. Simonis, Heavy hadron spectroscopy and the bag model, Lith. J. Phys. 49, 19 (2009).

[32] S. M. Ikhdair and R. Sever, $B_{c}$ and heavy meson spectroscopy in the local approximation of the Schrodinger equation with relativistic kinematics, Int. J. Mod. Phys. A 20, 4035 (2005).

[33] A. Abd El-Hady, M. A. K. Lodhi, and J. P. Vary, $B_{c}$ mesons in a Bethe-Salpeter model, Phys. Rev. D 59, 094001 (1999).

[34] L. Motyka and K. Zalewski, Mass spectra and leptonic decay widths of heavy quarkonia, Eur. Phys. J. C 4, 107 (1998).

[35] C. T. H. Davies, K. Hornbostel, G. P. Lepage, A. J. Lidsey, J. Shigemitsu, and J. H. Sloan, B(c) spectroscopy from lattice QCD, Phys. Lett. B 382, 131 (1996).

[36] N. Mathur, M. Padmanath, and S. Mondal, Precise Predictions of Charmed-Bottom Hadrons from Lattice QCD, Phys. Rev. Lett. 121, 202002 (2018).

[37] R. J. Dowdall, C. T. H. Davies, T. C. Hammant, and R. R. Horgan, Precise heavy-light meson masses and hyperfine splittings from lattice QCD including charm quarks in the sea, Phys. Rev. D 86, 094510 (2012).

[38] E. B. Gregory, C. T. H. Davies, E. Follana, E. Gamiz, I. D. Kendall, G. P. Lepage, H. Na, J. Shigemitsu, and K. Y. Wong, A Prediction of the $B_{c}^{*}$ Mass in Full Lattice QCD, Phys. Rev. Lett. 104, 022001 (2010).

[39] I. F. Allison, C. T. H. Davies, A. Gray, A. S. Kronfeld, P. B. Mackenzie, and J. N. Simone (HPQCD, Fermilab Lattice, and UKQCD Collaborations), Mass of the $B_{c}$ Meson in Three-Flavor Lattice QCD, Phys. Rev. Lett. 94, 172001 (2005).

[40] K. M. Cheung, $B_{c}$ Mesons Production at Hadron Colliders by Heavy Quark Fragmentation, Phys. Rev. Lett. 71, 3413 (1993).

[41] E. Braaten, S. Fleming, and T. C. Yuan, Production of heavy quarkonium in high-energy colliders, Annu. Rev. Nucl. Part. Sci. 46, 197 (1996). 
[42] K. M. Cheung and T. C. Yuan, Heavy quark fragmentation functions for $d$ wave quarkonium and charmed beauty mesons, Phys. Rev. D 53, 3591 (1996).

[43] K. M. Cheung and T. C. Yuan, Hadronic production of S wave and $\mathrm{P}$ wave charmed beauty mesons via heavy quark fragmentation, Phys. Rev. D 53, 1232 (1996).

[44] A. V. Berezhnoy, V. V. Kiselev, and A. K. Likhoded, Hadronic production of $\mathrm{S}$ and $\mathrm{P}$ wave states of $\bar{b} c$ quarkonium, Z. Phys. A 356, 79 (1996).

[45] C. H. Chang, J. X. Wang, and X. G. Wu, Hadronic production of the P-wave excited $B_{c}$-states $B_{c J, L=1}^{*}$, Phys. Rev. D 70, 114019 (2004).

[46] C. H. Chang, C. F. Qiao, J. X. Wang, and X. G. Wu, The Color-octet contributions to P-wave $B_{c}$ meson hadroproduction, Phys. Rev. D 71, 074012 (2005).

[47] Q. L. Liao, Y. Deng, Y. Yu, G. C. Wang, and G. Y. Xie, Heavy $P$-wave quarkonium production via Higgs decays, Phys. Rev. D 98, 036014 (2018).

[48] K. He, H. Y. Bi, R. Y. Zhang, X. Z. Li, and W. G. Ma, P-wave excited $B_{c}^{* *}$ meson photoproduction at the LHeC, J. Phys. G 45, 055005 (2018).

[49] Q. L. Liao, Y. Yu, Y. Deng, G. Y. Xie, and G. C. Wang, Excited heavy quarkonium production via $Z^{0}$ decays at a high luminosity collider, Phys. Rev. D 91, 114030 (2015).

[50] Q. L. Liao and G. Y. Xie, Heavy quarkonium wave functions at the origin and excited heavy quarkonium production via top quark decays at the LHC, Phys. Rev. D 90, 054007 (2014).

[51] Q. L. Liao, X. G. Wu, J. Jiang, Z. Yang, and Z. Y. Fang, Heavy quarkonium production at LHC through $W$ boson decays, Phys. Rev. D 85, 014032 (2012).

[52] Z. Yang, X. G. Wu, L. C. Deng, J. W. Zhang, and G. Chen, Production of the $P$-wave excited $B_{c}$-states through the $Z^{0}$ boson decays, Eur. Phys. J. C 71, 1563 (2011).

[53] C. H. Chang, J. X. Wang, and X. G. Wu, Production of $B_{c}$ or $\bar{B}_{c}$ meson and its excited states via $\bar{t}$ quark or $t$ quark decays, Phys. Rev. D 77, 014022 (2008).

[54] S. Patnaik, P. C. Dash, S. Kar, S. Patra, and N. Barik, Magnetic dipole transitions of $B_{c}$ and $B_{c}^{*}$ mesons in the relativistic independent quark model, Phys. Rev. D 96, 116010 (2017); Erratum 99, 019901(E) (2019).

[55] V. Simonis, Magnetic properties of ground-state mesons, Eur. Phys. J. A 52, 90 (2016).

[56] T. Wang, Y. Jiang, W. L. Ju, H. Yuan, and G. L. Wang, The electromagnetic decays of $B_{c}(2 S)$, J. High Energy Phys. 03 (2016) 209.

[57] Z. G. Wang, The radiative decays $B_{c}^{* \pm} \rightarrow B_{c}^{ \pm} \gamma$ with QCD sum rules, Eur. Phys. J. C 73, 2559 (2013).

[58] D. Ebert, R. N. Faustov, and V. O. Galkin, Radiative M1 decays of heavy light mesons in the relativistic quark model, Phys. Lett. B 537, 241 (2002).

[59] J. Ferretti and E. Santopinto, Open-flavor strong decays of open-charm and open-bottom mesons in the ${ }^{3} P_{0}$ model, Phys. Rev. D 97, 114020 (2018).

[60] V. V. Kiselev, Decay of $B_{c}^{*+}(3 S) \rightarrow B^{+} D^{0}$, Phys. Lett. B 390, 427 (1997).

[61] W. J. Deng, H. Liu, L. C. Gui, and X. H. Zhong, Charmonium spectrum and their electromagnetic transitions with higher multipole contributions, Phys. Rev. D 95, 034026 (2017).
[62] W. J. Deng, H. Liu, L. C. Gui, and X. H. Zhong, Spectrum and electromagnetic transitions of bottomonium, Phys. Rev. D 95, 074002 (2017).

[63] L. Micu, Decay rates of meson resonances in a quark model, Nucl. Phys. B10, 521 (1969).

[64] A. Le Yaouanc, L. Oliver, O. Pene, and J. C. Raynal, Naive quark pair creation model of strong interaction vertices, Phys. Rev. D 8, 2223 (1973).

[65] A. Le Yaouanc, L. Oliver, O. Pene, and J.-C. Raynal, Naive quark pair creation model and baryon decays, Phys. Rev. D 9, 1415 (1974).

[66] T. Barnes, S. Godfrey, and E. S. Swanson, Higher charmonia, Phys. Rev. D 72, 054026 (2005).

[67] C. H. Cai and L. Li, Radial equation of bound state and binding energies of $\Xi^{-}$hypernuclei, Chin. Phys. C 27, 1005 (2003).

[68] L. Y. Xiao, K. L. Wang, Q. F. Lü, X. H. Zhong, and S. L. Zhu, Strong and radiative decays of the doubly charmed baryons, Phys. Rev. D 96, 094005 (2017).

[69] K. L. Wang, L. Y. Xiao, X. H. Zhong, and Q. Zhao, Understanding the newly observed $\Omega_{c}$ states through their decays, Phys. Rev. D 95, 116010 (2017).

[70] Q. F. Lü, K. L. Wang, L. Y. Xiao, and X. H. Zhong, Mass spectra and radiative transitions of doubly heavy baryons in a relativized quark model, Phys. Rev. D 96, 114006 (2017).

[71] K. L. Wang, Y. X. Yao, X. H. Zhong, and Q. Zhao, Strong and radiative decays of the low-lying $S$ - and $P$-wave singly heavy baryons, Phys. Rev. D 96, 116016 (2017).

[72] Y.X. Yao, K. L. Wang, and X. H. Zhong, Strong and radiative decays of the low-lying $D$-wave singly heavy baryons, Phys. Rev. D 98, 076015 (2018).

[73] S. J. Brodsky and J. R. Primack, The Electromagnetic interactions of composite systems, Ann. Phys. (N.Y.) 52, 315 (1969).

[74] Z. P. Li, H.X. Ye, and M. H. Lu, A unified approach to pseudoscalar meson photoproductions off nucleons in the quark model, Phys. Rev. C 56, 1099 (1997).

[75] Q. Zhao, J. S. Al-Khalili, Z. P. Li, and R. L. Workman, Pion photoproduction on the nucleon in the quark model, Phys. Rev. C 65, 065204 (2002).

[76] L. Y. Xiao, X. Cao, and X. H. Zhong, Neutral pion photoproduction on the nucleon in a chiral quark model, Phys. Rev. C 92, 035202 (2015).

[77] X. H. Zhong and Q. Zhao, $\eta$ photoproduction on the quasifree nucleons in the chiral quark model, Phys. Rev. C 84, 045207 (2011).

[78] X. H. Zhong and Q. Zhao, $\eta^{\prime}$ photoproduction on the nucleons in the quark model, Phys. Rev. C 84, 065204 (2011).

[79] M. Jacob and G.C. Wick, On the general theory of collisions for particles with spin, Ann. Phys. (N.Y.) 7, 404 (1959); 281, 774 (2000).

[80] L. C. Gui, L. S. Lu, Q. F. Lü, X. H. Zhong, and Q. Zhao, Strong decays of higher charmonium states into open-charm meson pairs, Phys. Rev. D 98, 016010 (2018).

[81] Q. F. Lü, T. T. Pan, Y. Y. Wang, E. Wang, and D. M. Li, Excited bottom and bottom-strange mesons in the quark model, Phys. Rev. D 94, 074012 (2016).

[82] D. M. Li, P. F. Ji, and B. Ma, The newly observed opencharm states in quark model, Eur. Phys. J. C 71, 1582 (2011). 
[83] M. Tanabashi et al. (Particle Data Group), Review of particle physics, Phys. Rev. D 98, 030001 (2018).

[84] E.S. Ackleh, T. Barnes, and E. S. Swanson, On the mechanism of open flavor strong decays, Phys. Rev. D 54, 6811 (1996).

[85] T. Barnes, N. Black, and P. R. Page, Strong decays of strange quarkonia, Phys. Rev. D 68, 054014 (2003).
[86] S. Godfrey, K. Moats, and E. S. Swanson, $B$ and $B_{s}$ Meson Spectroscopy, Phys. Rev. D 94, 054025 (2016).

[87] S. Godfrey and K. Moats, Properties of excited charm and charm-strange mesons, Phys. Rev. D 93, 034035 (2016).

[88] F. E. Close and E. S. Swanson, Dynamics and decay of heavy-light hadrons, Phys. Rev. D 72, 094004 (2005). 